\title{
Theory of Nonlinear Guided Electromagnetic Waves in a Plane Two-Layered Dielectric Waveguide
}

\author{
Valeria Yu. Kurseeva and Dmitry V. Valovik \\ Department of Mathematics and Supercomputing, Penza State University, 40 Krasnaya Street, Penza 440026, Russia \\ Correspondence should be addressed to Valeria Yu. Kurseeva; lynxbax@mail.ru
}

Received 23 July 2017; Accepted 19 September 2017; Published 26 October 2017

Academic Editor: Ivan D. Rukhlenko

Copyright (c) 2017 Valeria Yu. Kurseeva and Dmitry V. Valovik. This is an open access article distributed under the Creative Commons Attribution License, which permits unrestricted use, distribution, and reproduction in any medium, provided the original work is properly cited.

\begin{abstract}
Propagation of transverse electric electromagnetic waves in a homogeneous plane two-layered dielectric waveguide filled with a nonlinear medium is considered. The original wave propagation problem is reduced to a nonlinear eigenvalue problem for an equation with discontinuous coefficients. The eigenvalues are propagation constants (PCs) of the guided waves that the waveguide supports. The existence of PCs that do not have linear counterparts and therefore cannot be found with any perturbation method is proven. PCs without linear counterparts correspond to a novel propagation regime that arises due to the nonlinearity. Numerical results are also presented; the comparison between linear and nonlinear cases is made.
\end{abstract}

\section{Introduction}

Theory of electromagnetic wave propagation in regular (planar, cylindrical, etc.) waveguides filled with linear dielectrics traditionally attracts attention [1-4]. This theory is interesting due to several reasons: first, such problems describe real physical processes that are of importance for applications; second, from the mathematical point of view, this theory is an affluent source of sophisticated and interesting mathematical problems.

Theory of electromagnetic waves in nonlinear media has also attracted attention for decades [5-12]. There are a lot of topics in this field, for example, electromagnetic wave propagation in self-focusing and self-defocusing media, higher harmonic generation (especially second and third), and Raman scattering $[6,8,10,13]$.

In the theory of nonlinear electromagnetic wave propagation, the most advanced results can be found for the case of monochromatic polarised (TE and TM) waves in planar layered dielectric waveguides. From the mathematical standpoint, similar problems for circle cylindrical waveguides are much more complicated. To the best of our knowledge, the first rigorous formulation of TE and TM wave propagation in plane and circle cylindrical waveguides with nonlinear filling had been proposed in [14], and since then these and similar problems have been studied very intensively [7, 10, 15-25]. Nevertheless, key results in the cases of TE and TM wave propagation in a layer with Kerr nonlinearity have been found only recently [23-25].

It is worth noting that the development of the wave propagation theory in a single layer is a first step towards studying stratified (or multilayered) waveguide structures. Layered and periodic waveguides are of special interest for optical guiding industry. Since such structures play an important role in a number of applications in optics, then they compel attention of researchers [26-35].

This paper focuses on the problem of monochromatic TE wave propagation in a plane two-layered dielectric waveguide $\Sigma$ filled with Kerr media. The guided wave harmonically depends on one of the longitudinal coordinates and decays along the transverse coordinate. Perfectly conducted wall is located on one of the waveguide boundaries; on the opposite side, the waveguide is open and the half-space is filled with a homogeneous isotropic nonmagnetic medium having constant permittivity. We apply the approach developed in [36]. From the mathematical point of view, the problem under investigation is a nonlinear eigenvalue problem for an ordinary nonlinear autonomous differential equation of the 


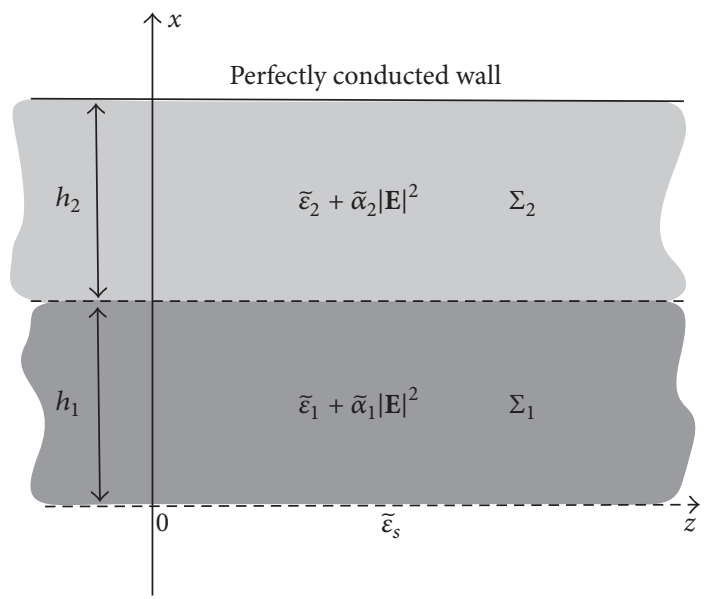

FIGURE 1: Geometry of the problem. Here $\widetilde{\varepsilon}_{i}=\varepsilon_{0} \varepsilon_{i}, \widetilde{\varepsilon}_{s}=\varepsilon_{0} \varepsilon_{s}$, and $\widetilde{\alpha}_{i}=\varepsilon_{0} \alpha_{i}$.

second order with discontinuous coefficients and boundary and transmission conditions followed from electromagnetic theory. Eigenvalues of the problem are propagation constants (PCs) of eigenwaves of the waveguide. The PCs are solutions to the so-called dispersion equation (DE). We derive the $\mathrm{DE}$ in the general case. If one of the layers is nonlinear and the other one is linear, then the DE can be studied in detail $[23,36]$.

\section{Statement of the Problem}

We consider the propagation of a monochromatic TE wave $(\mathbf{E}, \mathbf{H}) e^{-i \omega t}$, where $\omega$ is the circular frequency, in a lossless two-layered plane dielectric waveguide $\Sigma=\Sigma_{1} \cup \Sigma_{2}$, where

$$
\begin{aligned}
& \Sigma_{1}=\left\{(x, y, z): 0 \leqslant x<h_{1},(y, z) \in \mathbb{R}^{2}\right\}, \\
& \Sigma_{2}=\left\{(x, y, z): h_{1} \leqslant x \leqslant h_{1}+h_{2},(y, z) \in \mathbb{R}^{2}\right\} .
\end{aligned}
$$

The TE wave is described as follows:

$$
\begin{aligned}
& \mathbf{E}=\left(0, E_{y}(x) e^{i \gamma z}, 0\right)^{\top}, \\
& \mathbf{H}=\left(H_{x}(x) e^{i \gamma z}, 0, H_{z}(x) e^{i \gamma z}\right)^{\top},
\end{aligned}
$$

where $\mathbf{E}$ and $\mathbf{H}$ are the complex amplitudes [14] and $\gamma$ is an unknown real propagation constant (spectral parameter).

In the half-space $x<0$, the permittivity is constant and is equal to $\widetilde{\varepsilon}_{s}=\varepsilon_{0} \varepsilon_{s}$, where $\varepsilon_{s} \geqslant 1$ and $\varepsilon_{0}>0$ is the permittivity of free space. There are no sources in the entire space. Everywhere, $\mu=\mu_{0}$, where $\mu_{0}$ is the permeability of free space.

The waveguide $\Sigma$ is characterised by the permittivity $\widetilde{\varepsilon}=$ $\varepsilon_{0} \varepsilon$, where

$$
\varepsilon= \begin{cases}\varepsilon_{1}+\alpha_{1}|\mathbf{E}|^{2}, & (x, y, z) \in \Sigma_{1}, \\ \varepsilon_{2}+\alpha_{2}|\mathbf{E}|^{2}, & (x, y, z) \in \Sigma_{2}\end{cases}
$$

and $\alpha_{1}, \alpha_{2}>0$ (see Figure 1). In what follows, we assume that $\varepsilon_{s}<\varepsilon_{1}<\varepsilon_{2}$ are real constants. There is a perfectly conducted wall $\sigma$ at the boundary $x=h_{1}+h_{2}$.
Complex amplitudes (2) satisfy Maxwell's equations,

$$
\begin{aligned}
& \operatorname{rot} \mathbf{H}=-i \omega \widetilde{\varepsilon} \mathbf{E}, \\
& \operatorname{rot} \mathbf{E}=i \omega \mu_{0} \mathbf{H},
\end{aligned}
$$

and decay as $O\left(|x|^{-1}\right)$ when $x \rightarrow-\infty$; tangential components of the fields are continuous on the boundaries $x=0$ and $x=$ $h_{1}$; tangential component of the electric field vanishes on the boundary $x=h_{1}+h_{2}$. It is assumed that the value $\left.E_{y}\right|_{x=0} \neq 0$ is prescribed.

If it does not lead to misunderstanding, the explicit dependence on $x$ and $\gamma$ is omitted.

Substituting (2) into (4), one gets

$$
\begin{aligned}
i \gamma H_{x}(x)-H_{z}^{\prime}(x) & =-i \omega \varepsilon_{0} \varepsilon E_{y}(x), \\
-i \gamma E_{y}(x) & =i \omega \mu_{0} H_{x}(x), \\
E_{y}^{\prime}(x) & =i \omega \mu_{0} H_{z}(x) .
\end{aligned}
$$

Let $k_{0}^{2}=\omega^{2} \mu_{0} \varepsilon_{0}$. Expressing $H_{x}$ and $H_{z}$ from the second and third equations in (5) and substituting the results into the first equation, one obtains

$$
E_{y}^{\prime \prime}=\left(\gamma^{2}-k_{0}^{2} \varepsilon\right) E_{y} .
$$

Denoting $E_{y}$ by $y_{1}$ and $y_{2}$ in the layers $\Sigma_{1}$ and $\Sigma_{2}$, respectively, one obtains the equations

$$
\begin{aligned}
& y_{1}^{\prime \prime}=-\left(\kappa_{1}^{2}+\beta_{1} y_{1}^{2}\right) y_{1}, \\
& y_{1} \equiv y_{1}(x ; \gamma), \quad x \in\left[0, h_{1}\right], \\
& y_{2}^{\prime \prime}=-\left(\kappa_{2}^{2}+\beta_{2} y_{2}^{2}\right) y_{2}, \\
& y_{2} \equiv y_{2}(x ; \gamma), \quad x \in\left[h_{1}, h_{1}+h_{2}\right],
\end{aligned}
$$

where $\kappa_{i}^{2}:=k_{0}^{2} \varepsilon_{i}-\gamma^{2}$ and $\beta_{i}:=k_{0}^{2} \alpha_{i}(i=1,2) ; \kappa_{i}^{2}$ are not necessarily positive.

Equation (6) is linear in the half-space $x<0$. Taking into account conditions at infinity, one obtains its solution in the form

$$
E_{y}(x)=A e^{\kappa_{s} x}
$$

where $\kappa_{s}^{2}:=\gamma^{2}-k_{0}^{2} \varepsilon_{s}>0$. This solution results in the condition $\gamma^{2}>k_{0}^{2} \varepsilon_{s}$.

The continuity condition for the tangential field components results in the continuity of $E_{y}$ and $E_{y}^{\prime}$ at $x=0$ and $x=h_{1}$. Using solution (9) and the continuity of $E_{y}$ and $E_{y}^{\prime}$, one obtains $y_{1}(0)=A$ and $y_{1}^{\prime}(0)=\kappa_{s} A$. Since $E_{y}$ corresponds to the tangential component of the electric field, then it vanishes at $x=h_{1}+h_{2}$. In view of this, one gets the following conditions:

$$
\begin{array}{r}
y_{1}^{\prime}(0)-\kappa_{s} y_{1}(0)=0, \\
y_{2}\left(h_{1}+h_{2}\right)=0, \\
y_{1}\left(h_{1}-0\right)-y_{2}\left(h_{1}+0\right)=0, \\
y_{1}^{\prime}\left(h_{1}-0\right)-y_{2}^{\prime}\left(h_{1}+0\right)=0,
\end{array}
$$


where $y_{1}(0)=A \neq 0$ is supposed to be known (without loss of generality, $A>0)$.

Thus, the original wave propagation problem is reduced to the problem $P\left(\beta_{1}, \beta_{2}\right)$, which is to determine PCs $\gamma=\widehat{\gamma}$, such that there exists a nontrivial function

$$
y(x ; \widehat{\gamma})= \begin{cases}y_{1}(x ; \widehat{\gamma}), & x \in\left[0, h_{1}\right] \\ y_{2}(x ; \widehat{\gamma}), & x \in\left[h_{1}, h_{1}+h_{2}\right],\end{cases}
$$

which satisfies (7)-(8), conditions (10)-(11), and

$$
y \in C^{1}\left[0, h_{1}+h_{2}\right] \cap C^{2}\left[0, h_{1}\right] \cap C^{2}\left[h_{1}, h_{1}+h_{2}\right] .
$$

The problem $P\left(\beta_{1}, \beta_{2}\right)$ can be treated as an eigenvalue problem for a nonlinear differential equation of the second order with discontinuous coefficients on a segment with mixed boundary conditions and transmission conditions at the point $x=h_{1}$.

\section{Linear Problem}

Here we consider the case $\alpha_{1}=\alpha_{2}=0$, which corresponds to the linear problem denoted by $P(0,0)$. In this case, $(7)$ and (8) are linear:

$$
\begin{aligned}
& y_{1}^{\prime \prime}(x)=-\kappa_{1}^{2} y_{1}(x), \quad x \in\left[0, h_{1}\right], \\
& y_{2}^{\prime \prime}(x)=-\kappa_{2}^{2} y_{2}(x), \quad x \in\left[h_{1}, h_{1}+h_{2}\right] .
\end{aligned}
$$
form:

Using (10), solutions to (14) are written in the following

(i) For $\gamma^{2}>k_{0}^{2} \varepsilon_{2}$,

$$
\begin{aligned}
& y_{1}=A\left(\frac{\kappa_{s}}{\widetilde{\kappa}_{1}} \sinh \left(\widetilde{\kappa}_{1} x\right)+\cosh \left(\widetilde{\kappa}_{1} x\right)\right), \\
& y_{2}=B \sinh \left(\widetilde{\kappa}_{2}\left(x-h_{1}-h_{2}\right)\right),
\end{aligned}
$$

where $\widetilde{\kappa}_{1}^{2}:=-\kappa_{1}^{2}$ and $\widetilde{\kappa}_{2}^{2}:=-\kappa_{2}^{2}$.

(ii) For $k_{0}^{2} \varepsilon_{1}<\gamma^{2}<k_{0}^{2} \varepsilon_{2}$,

$$
\begin{aligned}
& y_{1}=A\left(\frac{\kappa_{s}}{\widetilde{\kappa}_{1}} \sinh \left(\widetilde{\kappa}_{1} x\right)+\cosh \left(\widetilde{\kappa}_{1} x\right)\right), \\
& y_{2}=B \sin \left(\kappa_{2}\left(x-h_{1}-h_{2}\right)\right),
\end{aligned}
$$

where $\widetilde{\kappa}_{1}^{2}:=-\kappa_{1}^{2}$.

(iii) For $k_{0}^{2} \varepsilon_{s}<\gamma^{2}<k_{0}^{2} \varepsilon_{1}$,

$$
\begin{aligned}
& y_{1}=A\left(\frac{\kappa_{s}}{\kappa_{1}} \sin \left(\kappa_{1} x\right)+\cos \left(\kappa_{1} x\right)\right), \\
& y_{2}=B \sin \left(\kappa_{2}\left(x-h_{1}-h_{2}\right)\right) .
\end{aligned}
$$

Using solutions (15)-(17) and (11), the DE for the problem $P(0,0)$ is written in the following form:

(i) For $\gamma^{2}>k_{0}^{2} \varepsilon_{2}$

$$
\frac{\kappa_{s} \sinh \left(\widetilde{\kappa}_{1} h_{1}\right)+\widetilde{\kappa}_{1} \cosh \left(\widetilde{\kappa}_{1} h_{1}\right)}{\kappa_{s} \cosh \left(\widetilde{\kappa}_{1} h_{1}\right)+\widetilde{\kappa}_{1} \sinh \left(\widetilde{\kappa}_{1} h_{1}\right)}=-\frac{\widetilde{\kappa}_{1} \sinh \left(\widetilde{\kappa}_{2} h_{2}\right)}{\widetilde{\kappa}_{2} \cosh \left(\widetilde{\kappa}_{2} h_{2}\right)},
$$

where $\widetilde{\kappa}_{1}^{2}=-\kappa_{1}^{2}$ and $\widetilde{\kappa}_{2}^{2}=-\kappa_{2}^{2}$. (ii) For $k_{0}^{2} \varepsilon_{1}<\gamma^{2}<k_{0}^{2} \varepsilon_{2}$,

$$
\frac{\kappa_{s} \sinh \left(\widetilde{\kappa}_{1} h_{1}\right)+\widetilde{\kappa}_{1} \cosh \left(\widetilde{\kappa}_{1} h_{1}\right)}{\kappa_{s} \cosh \left(\widetilde{\kappa}_{1} h_{1}\right)+\widetilde{\kappa}_{1} \sinh \left(\widetilde{\kappa}_{1} h_{1}\right)}=-\frac{\widetilde{\kappa}_{1} \sin \left(\kappa_{2} h_{2}\right)}{\kappa_{2} \cos \left(\kappa_{2} h_{2}\right)},
$$

where $\widetilde{\kappa}_{1}^{2}=-\kappa_{1}^{2}$.

(iii) For $k_{0}^{2} \varepsilon_{s}<\gamma^{2}<k_{0}^{2} \varepsilon_{1}$,

$$
\frac{\kappa_{s} \sin \left(\kappa_{1} h_{1}\right)+\kappa_{1} \cos \left(\kappa_{1} h_{1}\right)}{\kappa_{s} \cos \left(\kappa_{1} h_{1}\right)-\kappa_{1} \sin \left(\kappa_{1} h_{1}\right)}=-\frac{\kappa_{1} \sin \left(\kappa_{2} h_{2}\right)}{\kappa_{2} \cos \left(\kappa_{2} h_{2}\right)} .
$$

It is clear that (18) has no solutions. Indeed, the left-hand side is positive and right-hand side is negative. Thus, solutions to the linear problem satisfy the condition

$$
k_{0}^{2} \varepsilon_{s}<\gamma^{2}<k_{0}^{2} \varepsilon_{2}
$$

Let us consider (19). Introduce the function

$$
\begin{aligned}
F & \left(\gamma^{2}\right) \\
= & \kappa_{2} \cos \left(\kappa_{2} h_{2}\right)\left(\kappa_{s} \sinh \left(\widetilde{\kappa}_{1} h_{1}\right)+\widetilde{\kappa}_{1} \cosh \left(\widetilde{\kappa}_{1} h_{1}\right)\right) \\
& \quad+\widetilde{\kappa}_{1} \sin \left(\kappa_{2} h_{2}\right)\left(k_{s} \cosh \left(\widetilde{\kappa}_{1} h_{1}\right)+\widetilde{\kappa}_{1} \sinh \left(\widetilde{\kappa}_{1} h_{1}\right)\right) .
\end{aligned}
$$

Let $\sin \left(\kappa_{2} h_{2}\right)=0$; therefore $\gamma^{2}=k_{0}^{2} \varepsilon_{2}-\left(\pi n / h_{2}\right)^{2}=\gamma_{*}^{2}$, where $n \geqslant 1$ is an integer, such that $k_{0}^{2} \varepsilon_{2}-\left(\pi n / h_{2}\right)^{2}>k_{0}^{2} \varepsilon_{1}$ [see formula (19)]. Thus, one obtains

$$
F\left(\gamma_{*}^{2}\right)=\frac{\pi n}{h_{2}}(-1)^{n}\left(\kappa_{s} \sinh \left(\widetilde{\kappa}_{1} h_{1}\right)+\widetilde{\kappa}_{1} \cosh \left(\widetilde{\kappa}_{1} h_{1}\right)\right) .
$$

Choosing the lowest possible $n=1$, one gets $F\left(\gamma_{*}^{2}\right)<0$. The inequality $\varepsilon_{2}-\pi^{2} / k_{0}^{2} h_{2}^{2}>\varepsilon_{1}$ must be fulfilled.

Now let $\cos \left(\kappa_{2} h_{2}\right)=0$; therefore $\gamma^{2}=k_{0}^{2} \varepsilon_{2}-((\pi+$ $\left.2 \pi m) / 2 h_{2}\right)^{2}=\gamma^{* 2}$, where $m \geqslant 0$ is an integer, such that $k_{0}^{2} \varepsilon_{2}-\left((\pi+2 \pi m) / 2 h_{2}\right)^{2}>k_{0}^{2} \varepsilon_{1}$ [see formula (19)]. Thus, one obtains

$$
F\left(\gamma^{* 2}\right)=\widetilde{\kappa}_{1}(-1)^{m}\left(\kappa_{s} \cosh \left(\widetilde{\kappa}_{1} h_{1}\right)+\widetilde{\kappa}_{1} \sinh \left(\widetilde{\kappa}_{1} h_{1}\right)\right) .
$$

Choosing the lowest possible $m=0$, one gets $F\left(\gamma^{* 2}\right)>0$. The inequality $\varepsilon_{2}-\pi^{2} / 4 k_{0}^{2} h_{2}^{2}>\varepsilon_{1}$ must be fulfilled.

Since $F$ is continuous for $\gamma \in\left(\gamma_{*}, \gamma^{*}\right)$ and $F\left(\gamma_{*}^{2}\right) F\left(\gamma^{* 2}\right)<$ 0 , then there is $\tilde{\gamma} \in\left(\gamma_{*}, \gamma^{*}\right)$ such that $F(\widetilde{\gamma})=0$. The inequality

$$
h_{2}>\frac{\pi}{k_{0} \sqrt{\varepsilon_{2}-\varepsilon_{1}}}
$$

is a sufficient condition of existence of solutions to (19).

Let us pass to (20). Introduce the function

$$
\begin{aligned}
& F\left(\gamma^{2}\right) \\
& =\kappa_{2} \cos \left(\kappa_{2} h_{2}\right)\left(\kappa_{s} \sin \left(\kappa_{1} h_{1}\right)+\kappa_{1} \cos \left(\kappa_{1} h_{1}\right)\right) \\
& \quad+\kappa_{1} \sin \left(\kappa_{2} h_{2}\right)\left(\kappa_{s} \cos \left(\kappa_{1} h_{1}\right)-\kappa_{1} \sin \left(\kappa_{1} h_{1}\right)\right) .
\end{aligned}
$$


Let $\sin \left(\kappa_{2} h_{2}\right)=0$; therefore $\gamma^{2}=k_{0}^{2} \varepsilon_{2}-\left(\pi n / h_{2}\right)^{2}=\gamma_{*}^{2}$, where $n \geqslant 1$ is an integer, such that $k_{0}^{2} \varepsilon_{s}<k_{0}^{2} \varepsilon_{2}-\left(\pi n / h_{2}\right)^{2}<$ $k_{0}^{2} \varepsilon_{1}$ [see formula (20)]. Thus, one obtains

$$
\begin{aligned}
F\left(\gamma_{*}^{2}\right) & =\frac{\pi n}{h_{2}}(-1)^{n}\left(\kappa_{s} \sin \left(\kappa_{1} h_{1}\right)+\kappa_{1} \cos \left(\kappa_{1} h_{1}\right)\right) \\
& =\frac{\pi n}{h_{2}}(-1)^{n} \sqrt{\kappa_{1}^{2}+\kappa_{s}^{2}} \sin \left(\kappa_{1} h_{1}+\phi\right),
\end{aligned}
$$

where $\sin \phi=\kappa_{1} / \sqrt{\kappa_{1}^{2}+\kappa_{s}^{2}}$ and $\cos \phi=\kappa_{s} / \sqrt{\kappa_{1}^{2}+\kappa_{s}^{2}}$. Choosing the lowest possible $n=1$, one gets $F\left(\gamma_{*}^{2}\right)<0$ subject to $0<\kappa_{1} h_{1}+\phi<\pi$. Since the inequality $\varepsilon_{s}<$ $\varepsilon_{2}-\left(\pi / k_{0} h_{2}\right)^{2}<\varepsilon_{1}$ must be fulfilled, then $\pi / k_{0} \sqrt{\varepsilon_{2}-\varepsilon_{s}}<$ $h_{2}<\pi / k_{0} \sqrt{\varepsilon_{2}-\varepsilon_{1}}$.

Now let $\cos \left(\kappa_{2} h_{2}\right)=0$; therefore $\gamma^{2}=k_{0}^{2} \varepsilon_{2}-((\pi+$ $\left.2 \pi m) / 2 h_{2}\right)^{2}=\gamma^{* 2}$, where $m \geqslant 0$ is an integer, such that $k_{0}^{2} \varepsilon_{s}<k_{0}^{2} \varepsilon_{2}-\left((\pi+2 \pi m) / 2 h_{2}\right)^{2}<k_{0}^{2} \varepsilon_{1}$ [see formula (20)]. Thus, one obtains

$$
\begin{aligned}
F\left(\gamma^{* 2}\right) & =\kappa_{1}(-1)^{m}\left(\kappa_{s} \cos \left(\kappa_{1} h_{1}\right)-\kappa_{1} \sin \left(\kappa_{1} h_{1}\right)\right) \\
& =\kappa_{1}(-1)^{m} \sqrt{\kappa_{1}^{2}+\kappa_{s}^{2}} \cos \left(\kappa_{1} h_{1}+\phi\right) .
\end{aligned}
$$

Choosing the lowest possible $m=0$, one gets $F\left(\gamma^{* 2}\right)>0$ subject to $\kappa_{1} h_{1}+\phi<\pi / 2$. Since the inequality $\varepsilon_{s}<\varepsilon_{2}-$ $\left(\pi / 2 k_{0} h_{2}\right)^{2}<\varepsilon_{1}$ must be fulfilled, then $\pi / 2 k_{0} \sqrt{\varepsilon_{2}-\varepsilon_{s}}<h_{2}<$ $\pi / 2 k_{0} \sqrt{\varepsilon_{2}-\varepsilon_{1}}$.

It follows from the above that the inequalities

$$
\begin{aligned}
\frac{\pi}{k_{0} \sqrt{\varepsilon_{2}-\varepsilon_{s}}} & <h_{2}<\frac{\pi}{2 k_{0} \sqrt{\varepsilon_{2}-\varepsilon_{1}}}, \\
h_{1} & <\frac{1}{\kappa_{1}}\left(\frac{\pi}{2}-\phi\right)
\end{aligned}
$$

give a sufficient condition of existence of solutions to (20).

Thus, we formulate the following.

Statement 1. The problem $P(0,0)$ does not have more than a finite number of PCs $\widetilde{\gamma}_{i}, i=\overline{1, l}$. For any $i=\overline{1, l}$, it is true that $k_{0}^{2} \varepsilon_{s}<\widetilde{\gamma}_{i}^{2}<k_{0}^{2} \varepsilon_{2}$.

Proof. The existence of solutions to (19) and (20) subject to conditions (25) and (29), respectively, results from the analysis given above. These conditions are only sufficient. It is also clear how conditions (25) and (29) change when the number of solutions increases.

The functions $F$ depend analytically on $\gamma$. Since $\gamma$ belongs to a finite interval [see formula (21)], then each of (19) and (20) does not have more than a finite number of isolated solutions inside the interval.

\section{Nonlinear Problem: Dispersion Equations and Theorem of Equivalence}

In the following, we need auxiliary results given below by Statements 2 and 3.
Statement 2. The Cauchy problem for (7) with initial data

$$
\begin{aligned}
& y_{1}(0)=A, \\
& y_{1}^{\prime}(0)=A \kappa_{s},
\end{aligned}
$$

where $A>0$ and $\kappa_{s}=\sqrt{\gamma^{2}-k_{0}^{2} \varepsilon_{s}}>0$ are constants, has a unique continuous solution $y_{1} \equiv y_{1}(x ; \gamma)$ defined globally on $\left[0, x^{*}\right]$, where $x^{*}>0$ is an arbitrary real point. This solution depends continuously on $\gamma$ for all $\gamma^{2}>k_{0}^{2} \varepsilon_{s}$.

Proof. First integral of (7) takes the form

$$
y_{1}^{\prime 2}+\kappa_{1}^{2} y_{1}^{2}+\frac{1}{2} \beta_{1} y_{1}^{4}=C_{1}
$$

where, using conditions (30), one finds

$$
C_{1}=\left(k_{0}^{2} \varepsilon_{1}-k_{0}^{2} \varepsilon_{s}+\frac{1}{2} \beta_{1} A^{2}\right) A^{2} .
$$

It is clear that $C_{1}$ does not depend on $\gamma$, and $C_{1}>0$ if $\beta_{1} \geqslant 0$. Introduce new variables:

$$
\begin{aligned}
& \tau_{1}(x)=y_{1}^{2}(x), \\
& \eta_{1}(x)=\frac{y_{1}^{\prime}(x)}{y_{1}(x)} .
\end{aligned}
$$

Equation (7) can be rewritten as a system:

$$
\begin{aligned}
& \tau_{1}^{\prime}=2 \tau_{1} \eta_{1}, \\
& \eta_{1}^{\prime}=-\left(\eta_{1}^{2}+\kappa_{1}^{2}+\beta_{1} \tau_{1}\right) .
\end{aligned}
$$

First integral (31) takes the form

$$
\frac{1}{2} \beta_{1} \tau_{1}^{2}+\left(\eta_{1}^{2}+\kappa_{1}^{2}\right) \tau_{1}=C_{1} .
$$

Solving (35) with respect to $\tau_{1}$, taking into account the fact that $\tau_{1} \geqslant 0$, and substituting the result into the right-hand side of the second equation in (34), one obtains

$$
\eta_{1}^{\prime}=-w_{1}\left(\eta_{1} ; \gamma\right)
$$

where $w_{1}\left(\eta_{1} ; \gamma\right)=\sqrt{\left(\eta_{1}^{2}+\kappa_{1}^{2}\right)^{2}+2 \beta_{1} C_{1}}$ and the radicand is positive for all real $\eta_{1}$ and $\gamma$.

Using conditions (30), one finds

$$
\eta_{1}(0)=\kappa_{s}>0
$$

Since $\eta_{1}^{\prime}<0, \eta_{1}$ monotonically decreases for $x>0$. In the general case, $y_{1}(x)$ can have zeros at some points on the interval $\left(0, x^{*}\right)$. Suppose that $y_{1}(x)$ has $n_{1}$ zeros $x_{1}, \ldots, x_{n_{1}} \in$ $\left(0, x^{*}\right)$. Then $\eta_{1}$ has $n_{1}$ break points $x_{1}, \ldots, x_{n_{1}} \in\left(0, x^{*}\right)$. If $n_{1}=0$, then $y_{1}(x)$ does not become zero for any $x \in\left[0, x^{*}\right]$ and, therefore, $\eta_{1}$ is continuous for $x \in\left[0, x^{*}\right]$. It is clear that $y_{1}^{\prime}\left(x_{i}\right) \neq 0$ for all $i=\overline{1, n_{1}}$. Formula (36) implies that

$$
\begin{aligned}
& \eta_{1}\left(x_{i}-0\right)=-\infty, \\
& \eta_{1}\left(x_{i}+0\right)=+\infty,
\end{aligned}
$$

$$
i=\overline{1, n_{1}} .
$$


Thereby, solutions to (36) are sought on each of the intervals $\left[0, x_{1}\right),\left(x_{1}, x_{2}\right), \ldots,\left(x_{n_{1}}, x^{*}\right)$ :

$$
\begin{aligned}
& \int_{\eta(x)}^{\eta\left(x_{1}-0\right)} \frac{d s}{w_{1}(s ; \gamma)}=x+c_{0}, \quad 0 \leqslant x<x_{1} ; \\
& -\int_{\eta\left(x_{i}+0\right)}^{\eta(x)} \frac{d s}{w_{1}(s ; \gamma)}=x+c_{i}, \\
& \quad x_{i}<x<x_{i+1}, \quad i=\overline{0, n_{1}-1} ; \\
& -\int_{\eta\left(x_{n_{1}}+0\right)}^{\eta(x)} \frac{d s}{w_{1}(s ; \gamma)}=x+c_{n_{1}}, \quad x_{n_{1}}<x<x^{*} .
\end{aligned}
$$

Substituting $x=0, x=x_{i+1}-0$, and $x=x^{*}$ into the first, second, and third equations, respectively, in (39), one determines

$$
\begin{aligned}
c_{0} & =\int_{\eta(0)}^{\eta\left(x_{1}-0\right)} \frac{d s}{w_{1}(s ; \gamma)} ; \\
c_{i} & =-\int_{\eta\left(x_{i}+0\right)}^{\eta\left(x_{i+1}-0\right)} \frac{d s}{w_{1}(s ; \gamma)}-x_{i+1}, \quad i=\overline{1, n_{1}-1} ; \\
c_{n_{1}} & =-\int_{\eta\left(x_{n_{1}}+0\right)}^{\eta\left(x^{*}\right)} \frac{d s}{w_{1}(s ; \gamma)}-x^{*} .
\end{aligned}
$$
form:

Using the found $c_{i}$, one can rewrite (39) in the following

$$
\begin{aligned}
& \int_{\eta(x)}^{\eta\left(x_{1}-0\right)} \frac{d s}{w_{1}(s ; \gamma)}=x+\int_{\eta(0)}^{\eta\left(x_{1}-0\right)} \frac{d s}{w_{1}(s ; \gamma)}, \\
& 0 \leqslant x<x_{1} \\
& -\int_{\eta\left(x_{i}+0\right)}^{\eta(x)} \frac{d s}{w_{1}(s ; \gamma)}=x-\int_{\eta\left(x_{i}+0\right)}^{\eta\left(x_{i+1}-0\right)} \frac{d s}{w_{1}(s ; \gamma)}-x_{i+1} \text {, } \\
& x_{i}<x<x_{i+1}, i=\overline{1, n_{1}-1} \text {; } \\
& -\int_{\eta\left(x_{n_{1}}+0\right)}^{\eta(x)} \frac{d s}{w_{1}(s ; \gamma)}=x-\int_{\eta\left(x_{n_{1}}+0\right)}^{\eta\left(x^{*}\right)} \frac{d s}{w_{1}(s ; \gamma)}-x^{*}, \\
& x_{n_{1}}<x<x^{*} .
\end{aligned}
$$

By substituting $x=x_{1}-0, x=x_{i}+0$, and $x=x_{n}+0$ into the first, second, and third equations, respectively, of previous equation, one obtains

$$
\begin{aligned}
& 0=x_{1}+\int_{\eta(0)}^{\eta\left(x_{1}-0\right)} \frac{d s}{w_{1}(s ; \gamma)}, \\
& 0=x_{i}-\int_{\eta\left(x_{i}+0\right)}^{\eta\left(x_{i+1}-0\right)} \frac{d s}{w_{1}(s ; \gamma)}-x_{i+1}, \quad i=\overline{1, n_{1}-1} \\
& 0=x_{n_{1}}-\int_{\eta\left(x_{n_{1}}+0\right)}^{\eta\left(x^{*}\right)} \frac{d s}{w_{1}(s ; \gamma)}-x^{*}
\end{aligned}
$$

Taking into account (37) and (38), one finds, from (42),

$$
\begin{aligned}
& 0<x_{1}=\int_{-\infty}^{\kappa_{s}} \frac{d s}{w_{1}(s ; \gamma)} \\
& 0<x_{i+1}-x_{i}=\int_{-\infty}^{+\infty} \frac{d s}{w_{1}(s ; \gamma)}, \quad i=\overline{1, n_{1}-1} \\
& 0<h_{1}-x_{n_{1}}=\int_{\eta\left(x^{*}\right)}^{+\infty} \frac{d s}{w_{1}(s ; \gamma)} .
\end{aligned}
$$

Formulas (43) give explicit expressions for distances between zeros of $y_{1}$. Moreover, since the left-hand sides in (43) are finite, the right-hand sides are also finite. Therefore, the improper integrals on the right-hand sides converge.

Summing up all the terms in (43), one gets

$$
\begin{aligned}
x_{1}+ & x_{2}-x_{1}+x_{3}-x_{2}+\cdots+x_{n_{1}-1}-x_{n_{1}-2}+x_{n_{1}} \\
& -x_{n_{1}-1}+x^{*}-x_{n_{1}} \\
= & \int_{-\infty}^{\kappa_{s}} w_{1} d s+\left(n_{1}-1\right) \int_{-\infty}^{+\infty} w_{1} d s+\int_{\eta\left(x^{*}\right)}^{+\infty} w_{1} d s .
\end{aligned}
$$

From the last formula, one finds

$$
\int_{\eta\left(x^{*}\right)}^{\kappa_{s}} w_{1} d s+n_{1} \int_{-\infty}^{+\infty} w_{1} d s=x^{*} .
$$

Formula (45) shows that the solution of the Cauchy problem to (7) with initial conditions (30) exists and is defined globally at any segment $\left[0, x^{*}\right]$. The uniqueness of this solution and its continuity with respect to $\gamma$ follows from smoothness of the right-hand side of (7) with respect to $y_{1}$ and $\gamma$ [37].

Let us consider the function $p(\gamma):=y_{1}^{\prime}\left(h_{1} ; \gamma\right) / y_{1}\left(h_{1} ; \gamma\right)$. Since the right-hand side of (7) depends on $\gamma$ analytically, the solution $y_{1} \equiv y_{1}(x ; \gamma)$ of the considered Cauchy problem depends on $\gamma$ analytically as well [38] and therefore $y_{1}$ and $y_{1}^{\prime}$ depend analytically on $\gamma$. Since $y_{1}$ and $y_{1}^{\prime}$ do not vanish simultaneously, $p \equiv p(\gamma)$ is an analytical function that can have only poles of the first order.

Passing to the limit $x^{*} \rightarrow h_{1}-0$ in (45), one gets

$$
\Phi_{1}\left(\gamma ; n_{1}, p\right) \equiv \int_{p}^{\kappa_{s}} w_{1} d s+n_{1} \int_{-\infty}^{+\infty} w_{1} d s=h_{1},
$$

where $n_{1}=0,1,2, \ldots$. We notice that if $p>\kappa_{s}$, then necessarily $n_{1} \geqslant 1$.

Statement 3. The Cauchy problem for (8) with initial data

$$
\begin{aligned}
& y_{2}\left(h_{1}+h_{2}\right)=0, \\
& y_{2}^{\prime}\left(h_{1}+h_{2}\right)=B,
\end{aligned}
$$

where $B$ is a real constant, has a unique continuous solution $y_{2} \equiv y_{2}(x ; \gamma)$ defined globally on $\left[x^{*}, h_{1}+h_{2}\right]$, where $x^{*}<$ $h_{1}+h_{2}$ is an arbitrary real point. This solution depends continuously on $\gamma$ for all $\gamma^{2}>k_{0}^{2} \varepsilon_{s}$. 
Proof. Now let us consider (8). First integral of (8) has the form

$$
y_{2}^{\prime 2}+\kappa_{2}^{2} y_{2}^{2}+\frac{1}{2} \beta_{2} y_{2}^{4}=C_{2}
$$

where, using condition (47), one calculates

$$
C_{2}=B^{2}>0
$$

where $B$ depends on $\gamma$. The positivity of $C_{2}$ is essential.

Introduce new variables:

$$
\begin{aligned}
& \tau_{2}(x):=y_{2}^{2}(x), \\
& \eta_{2}(x):=\frac{y_{2}^{\prime}(x)}{y_{2}(x)} .
\end{aligned}
$$

Equation (8) can be rewritten as a system:

$$
\begin{aligned}
& \tau_{2}^{\prime}=2 \tau_{2} \eta_{2}, \\
& \eta_{2}^{\prime}=-\left(\eta_{2}^{2}+\kappa_{2}^{2}+\beta_{2} \tau_{2}\right) .
\end{aligned}
$$

First integral (48) takes the form

$$
\frac{1}{2} \beta_{2} \tau_{2}^{2}+\left(\eta_{2}^{2}+\kappa_{2}^{2}\right) \tau_{2}=C_{1}
$$

Solving (52) with respect to $\tau_{2}$, taking into account the fact that $\tau_{2} \geqslant 0$, and substituting the result into the right-hand side of the second equation in (51), one obtains

$$
\eta_{2}^{\prime}=-w_{2}\left(\eta_{2} ; \gamma\right)
$$

where $w\left(\eta_{2} ; \gamma\right)=\sqrt{\left(\eta_{2}^{2}+\kappa_{2}^{2}\right)^{2}+2 \beta_{2} C_{2}}$ and the radicand is positive for all real $\eta_{2}$ and $\gamma$.

Using condition (47) and the fact that $\eta_{2}^{\prime}<0$, one finds

$$
\eta_{2}\left(h_{1}+h_{2}-0\right)=-\infty \text {. }
$$

Since $\eta_{2}^{\prime}<0, \eta_{2}$ monotonically decreases for $x \in\left(x^{*}, h_{1}+\right.$ $h_{2}$ ). However, $\eta_{2}$ is continuous if and only if $y_{2}(x)$ does not vanish for all $x \in\left(x^{*}, h_{1}+h_{2}\right)$. In the general case, $y_{2}(x)$ can have zeros at some points on the interval $\left(h_{1}, h_{1}+h_{2}\right)$. Suppose that $y_{2}(x)$ has $n_{2}$ zeros $x_{1}, \ldots, x_{n_{2}} \in\left(x^{*}, h_{1}+h_{2}\right)$. Then $\eta_{2}$ has $n_{2}$ break points $x_{1}, \ldots, x_{n_{2}} \in\left(x^{*}, h_{1}+h_{2}\right)$. It is clear that $y_{2}^{\prime}\left(x_{i}\right) \neq 0$ for all $i=\overline{1, n_{2}}$. Formula (53) implies that

$$
\begin{aligned}
& \eta_{2}\left(x_{i}-0\right)=-\infty, \\
& \eta_{2}\left(x_{i}+0\right)=+\infty, \\
& \quad i=\overline{1, n_{2}} .
\end{aligned}
$$

Thereby, solutions to (53) are sought on each of the intervals $\left(x^{*}, x_{1}\right),\left(x_{1}, x_{2}\right), \ldots,\left(x_{n_{2}}, h_{1}+h_{2}\right)$.

Using the same reasoning as in the proof of Statement 2, one obtains the expression

$$
\int_{-\infty}^{\eta\left(x^{*}\right)} \frac{d s}{w_{2}(s ; \gamma)}+n_{2} \int_{-\infty}^{+\infty} \frac{d s}{w_{2}(s ; \gamma)}=h_{2} .
$$

Formula (56) shows that the solution to the Cauchy problem for (8) with initial conditions (47) exists and is defined globally at any segment $\left[x^{*}, h_{1}+h_{2}\right]$. The uniqueness of this solution and its continuity with respect to $\gamma$ follows from smoothness of the right-hand side of (8) with respect to $y_{2}$ and $\gamma$ [37].

Passing to the limit $x^{*} \rightarrow h_{1}+0$ in (56), one gets

$$
\begin{aligned}
\Phi_{2}\left(\gamma ; n_{2}, p\right) & \equiv \int_{-\infty}^{p} \frac{d s}{w_{2}(s ; \gamma)}+n_{2} \int_{-\infty}^{+\infty} \frac{d s}{w_{2}(s ; \gamma)} \\
& =h_{2},
\end{aligned}
$$

where $n_{2}=0,1,2, \ldots$

The following theorem takes place.

Theorem 1 (of equivalence). The value $\widehat{\gamma}$ is a PC of the problem $P\left(\beta_{1}, \beta_{2}\right)$ if and only if there are integers $n_{1}=\widehat{n}_{1} \geqslant 0$ and $n_{2}=\widehat{n}_{2} \geqslant 0$ such that $\gamma=\widehat{\gamma}$ is a solution to the DE:

$$
\begin{aligned}
& \Phi_{1}\left(\gamma ; n_{1}, p\right)=h_{1}, \\
& \Phi_{2}\left(\gamma ; n_{2}, p\right)=h_{2}
\end{aligned}
$$

with certain $p=\widehat{p}$.

Proof. It follows from the derivation of expressions (46) and (57) that if $\gamma=\widehat{\gamma}$ is an eigenvalue of the problem $P\left(\beta_{1}, \beta_{2}\right)$, then it is a solution to system (58) with $n_{1}=\widehat{n}_{1}, n_{2}=\widehat{n}_{2}$, and $p=\widehat{p}$. Let us prove that each solution $\gamma=\widehat{\gamma}$ to system (58) is an eigenvalue.

Let system (58) have a solution $\gamma=\widehat{\gamma}$ with $n_{1}=\widehat{n}_{1}, n_{2}=$ $\widehat{n}_{2}, p=\widehat{p}$, and $y_{1}(0)=A$.

Consider the Cauchy problem for (7) with initial data (30), where $\kappa_{s}=\sqrt{\widehat{\gamma}^{2}-k_{0}^{2} \varepsilon_{s}}$. In accordance with Statement 2, its solution $y_{1} \equiv y_{1}(x, \widehat{\gamma})$ exists, is unique, and is defined for $x \in\left[0, h_{1}\right]$. At this step, we do not claim that $y_{1}^{\prime}\left(h_{1}\right) / y_{1}\left(h_{1}\right)=$ $\widehat{p}$. Using the found solution $y_{1}$ and formula (33), one determines the functions $\tau_{1}$ and $\eta_{1}$. It is clear that $\tau_{1}(0)=A^{2}$ and $\eta_{1}(0)=\kappa_{s}$.

Assuming that $\eta_{1}\left(h_{1}\right)=p_{1}<\widehat{p}$ and using the found $\tau_{1}$ and $\eta_{1}$, one obtains the expression

$$
\int_{p_{1}}^{\kappa_{s}} \frac{d s}{w_{1}(s ; \widehat{\gamma})}+\widehat{n}_{1} \int_{-\infty}^{+\infty} \frac{d s}{w_{1}(s ; \widehat{\gamma})}=h_{1},
$$

which corresponds to the first line in (58). We rewrite it in the form

$$
\int_{p_{1}}^{\widehat{p}} \frac{d s}{w_{1}(s ; \widehat{\gamma})}+\int_{\widehat{p}}^{\kappa_{s}} \frac{d s}{w_{1}(s ; \widehat{\gamma})}+\widehat{n}_{1} \int_{-\infty}^{+\infty} \frac{d s}{w_{1}(s ; \widehat{\gamma})}=h_{1} .
$$

Since $\gamma=\widehat{\gamma}$ satisfies the first line in (58) with $p=\widehat{p}$, then in the first line in (58) and in (60) the integrands coincide. Subtracting one from another, one obtains

$$
\int_{p_{1}}^{\widehat{p}} \frac{d s}{w_{1}(s ; \widehat{\gamma})}=0
$$


Due to the obvious estimates $\int_{-\infty}^{+\infty}\left(d s / w_{1}(s ; \widehat{\gamma})\right)>\int_{p_{1}}^{\widehat{p}}(d s /$ $\left.w_{1}(s ; \widehat{\gamma})\right)>0$, one obtains that (61) is fulfilled only if $p_{1}=\widehat{p}$. Therefore, the condition $\eta\left(h_{1}\right)=p_{1}<\widehat{p}$ is false. In the same way, it can be shown that the condition $\eta\left(h_{1}\right)=p_{1}>\widehat{p}$ is also false. Thus, $p_{1}=\widehat{p}$.

Now let us pass to the Cauchy problem for (8) with initial data (47), where $B \equiv B(\widehat{\gamma})$. In accordance with Statement 3 , its solution $y_{2} \equiv y_{2}(x, \widehat{\gamma})$ exists, is unique, and is defined globally for $x \in\left[h_{1}, h_{1}+h_{2}\right]$. In this case, using the continuity of $E_{y}$ and $E_{y}^{\prime}$, first integral (48), and (49), one finds that the quantity $B$ is determined from the equation

$$
B^{2}=y_{1}^{\prime 2}+\left(k_{0}^{2} \varepsilon_{2}-\widehat{\gamma}^{2}\right) y_{1}^{2}+\frac{1}{2} \beta_{2} y_{1}^{4}>0
$$

where $y_{1}$ and $y_{1}^{\prime}$ are calculated at $x=h_{1}$ with $\gamma=\widehat{\gamma}\left(y_{1}\right.$ and $y_{1}^{\prime}$ are already found).

At this step, we do not claim that $y_{2}^{\prime}\left(h_{1}\right) / y_{2}\left(h_{1}\right)=\widehat{p}$. Using the found solution $y_{2}$ and formula (50), one determines the functions $\tau_{2}$ and $\eta_{2}$.

We assume that $\eta_{2}\left(h_{1}\right)=p_{2}>\widehat{p}$. Using the found $\tau_{2}$ and $\eta_{2}$, one obtains the expression

$$
\int_{-\infty}^{p_{2}} \frac{d s}{w_{2}(s ; \widehat{\gamma})}+\widehat{n}_{2} \int_{-\infty}^{+\infty} \frac{d s}{w_{2}(s ; \widehat{\gamma})}=h_{2},
$$

which corresponds to the second line in (58). We rewrite it in the following form:

$$
\begin{aligned}
& \int_{-\infty}^{\widehat{p}} \frac{d s}{w_{2}(s ; \hat{\gamma})}+\int_{\widehat{p}}^{p_{2}} \frac{d s}{w_{2}(s ; \hat{\gamma})}+m_{2} \int_{-\infty}^{+\infty} \frac{d s}{w_{2}(s ; \hat{\gamma})} \\
& =h_{2} .
\end{aligned}
$$

Since $\gamma=\widehat{\gamma}$ satisfies the second line in (58) with $p=\widehat{p}$, then in the second line in (58) and in (64) the integrands coincide. Subtracting one from another, one obtains

$$
\int_{\widehat{p}}^{p_{2}} \frac{d s}{w_{2}(s ; \widehat{\gamma})}=0 .
$$

Due to the obvious estimates $\int_{-\infty}^{+\infty}\left(d s / w_{2}(s ; \widehat{\gamma})\right)>\int_{\widehat{p}}^{p_{2}}(d s /$ $\left.w_{2}(s ; \widehat{\gamma})\right)>0$, one obtains that $(65)$ is fulfilled only if $p_{2}=\widehat{p}$. Thus, the condition $\eta_{2}\left(h_{1}\right)=p_{2}>\widehat{p}$ is false. In the same way, it can be shown that the condition $\eta\left(h_{1}\right)=p_{2}<\widehat{p}$ is also false. Thus, $p_{2}=\widehat{p}$.

In other words, we have shown that the functions $y_{1}$ and $y_{2}$ satisfy (7) and (8) and conditions (10) and (11), and, therefore, the function

$$
y(x ; \widehat{\gamma})= \begin{cases}y_{1}(x ; \widehat{\gamma}), & x \in\left[0, h_{1}\right] \\ y_{2}(x ; \widehat{\gamma}), & x \in\left[h_{1}, h_{1}+h_{2}\right]\end{cases}
$$

is an eigenfunction of the problem $P\left(\beta_{1}, \beta_{2}\right)$ corresponding to the eigenvalue $\gamma=\widehat{\gamma}$.

\section{Solvability of the Nonlinear Problem}

Theorem 1 is derived for the general case, that is, $\beta_{1}, \beta_{2}>0$. The DE (58) can be studied theoretically and numerically. However, the existence of infinitely many PCs for the general case can be proven only if a special restriction is imposed on $B \equiv B(\gamma)$. This restriction establishes the behaviour of $B$ for big $\gamma$. To be more precise, $B(\gamma)$ must not decrease "too" rapid. As a matter of fact, such a restriction does not result from the physical formulation of the problem. For this reason below we study two simplified problems, where either $\beta_{1}$ or $\beta_{2}$ vanishes.

In this section, we use the following notation for the eigenvalues $\hat{\gamma}$ of problems $P\left(0, \beta_{2}\right)$ and $P\left(\beta_{1}, 0\right): \widehat{\gamma}_{i}$ means that all the eigenvalues are arranged in the ascending order; $\widehat{\gamma}(m)$ means that this eigenvalue is a solution of (57) with $n_{2}=m$ [for the problem $P\left(0, \beta_{2}\right)$ ] and (46) with $n_{1}=m$ [for the problem $\left.P\left(\beta_{1}, 0\right)\right]$.

5.1. Case $\beta_{1}=0$ and $\beta_{2} \neq 0$. If $\beta_{1}=0$ (or $\alpha_{1}=0$ ), solutions to (7) are found elementarily. This allows one to explicitly compute the quantity $p$, which is

$$
\begin{aligned}
p & =\kappa_{1} \frac{\kappa_{s} \cos \left(\kappa_{1} h_{1}\right)-\kappa_{1} \sin \left(\kappa_{1} h_{1}\right)}{\kappa_{s} \sin \left(\kappa_{1} h_{1}\right)+\kappa_{1} \cos \left(\kappa_{1} h_{1}\right)} \\
& =\kappa_{1} \frac{\kappa_{s}-\kappa_{1} \tan \left(\kappa_{1} h_{1}\right)}{\kappa_{1}+\kappa_{s} \tan \left(\kappa_{1} h_{1}\right)} .
\end{aligned}
$$

This expression (due to its analytical dependence on $\gamma$ ) can be used for $\kappa_{1}^{2} \geqslant 0$ as well as for $\kappa_{1}^{2} \leqslant 0$. Note that $p$ is a real value for all $\gamma^{2}>k_{0}^{2} \varepsilon_{s}$. Indeed, for $\gamma^{2}>k_{0}^{2} \varepsilon_{1}$, one has $\kappa_{1}=i \widetilde{\kappa}_{1}$, where $\widetilde{\kappa}_{1}^{2}=\gamma^{2}-k_{0}^{2} \varepsilon_{1}$, and then $\cos (i x)=\cosh (x)$ and $\sin (i x)=i \sinh (x)$; then

$$
\begin{aligned}
p & =\widetilde{\kappa}_{1} \frac{\kappa_{s} \cosh \left(\widetilde{\kappa}_{1} h_{1}\right)+\widetilde{\kappa}_{1} \sinh \left(\widetilde{\kappa}_{1} h_{1}\right)}{\kappa_{s} \sinh \left(\widetilde{\kappa}_{1} h_{1}\right)+\widetilde{\kappa}_{1} \cosh \left(\widetilde{\kappa}_{1} h_{1}\right)} \\
& =\kappa_{1} \frac{\kappa_{s}+\widetilde{\kappa}_{1} \tanh \left(\widetilde{\kappa}_{1} h_{1}\right)}{\widetilde{\kappa}_{1}+\kappa_{s} \tanh \left(\widetilde{\kappa}_{1} h_{1}\right)} .
\end{aligned}
$$

It can be checked that the substitution of the explicit expression for $p$ into the first equation of system (58) leads to the identity. Thus, in this case, system (58) reduces to the only equation

$$
\Phi_{2}\left(\gamma ; n_{2}, p\right)=h_{2}
$$

where $\Phi_{2}$ and $p$ are given by (57) and (67), respectively; $n_{2}=$ $0,1,2, \ldots$.

The solvability of the problem $P\left(0, \beta_{2}\right)$ is established by the following.

Theorem 2. For $\beta_{1}=0$, any $\beta_{2}>0$, and any fixed $A \neq 0$, the problem $P\left(0, \beta_{2}\right)$ has an infinite number of PCs $\widehat{\gamma}_{i}(i=1,2, \ldots)$ with the following properties:

(1) If $\widehat{\gamma}_{i}$ is the solution to $P\left(0, \beta_{2}\right)$, then $\widehat{\gamma}_{i}^{2} \in\left(k_{0}^{2} \varepsilon_{s},+\infty\right)$ and $\lim _{i \rightarrow \infty} \widehat{\gamma}_{i}^{2}=+\infty$.

(2) If the linear problem $P(0,0)$ has q solutions $\tilde{\gamma}_{1}<\tilde{\gamma}_{2}<$ $\cdots<\widetilde{\gamma}_{q}$, then there exists a constant $\beta_{0}>0$ such that, 
for any $\beta_{2}=\beta_{2}^{\prime}<\beta_{0}$, it is true that $\hat{\gamma}_{i}^{2} \in\left(k_{0}^{2} \varepsilon_{s}, k_{0}^{2} \varepsilon_{2}\right)$ and $\lim _{\beta_{1}^{\prime} \rightarrow 0} \widehat{\gamma}_{i}=\widetilde{\gamma}_{i}(i=\overline{1, q})$, where $\widehat{\gamma}_{1}, \widehat{\gamma}_{2}, \ldots, \widehat{\gamma}_{q}$ are first $q$ solutions to $P\left(0, \beta_{2}^{\prime}\right)$.

(3) If $\widehat{\gamma}_{i} \rightarrow \infty$, then $\max _{x \in\left(h_{1}, h_{1}+h_{2}\right)}\left|y_{2}\left(x, \widehat{\gamma}_{i}\right)\right| \rightarrow \infty$.

(4) For big $\hat{\gamma}$ and arbitrary small $\Delta>0$, the asymptotic two-sided inequality

$$
\begin{aligned}
(1- & \Delta) \frac{1}{h_{1}} \ln \frac{m \pi}{h_{2} A \sqrt{\beta_{2}}} \leqslant \hat{\gamma}(m) \\
& \leqslant(1+\Delta) \frac{1}{h_{1}} \ln \frac{\sqrt{2}(m+1) \pi}{h_{2} A \sqrt{\beta_{2}}}
\end{aligned}
$$

is valid.

Proof. Now let us study the behaviour of $C_{2} \equiv C_{2}(\gamma)$ with respect to $\gamma$. Explicit solution to (7) with initial data (30) has the form $y_{1}(x)=A\left(\left(\kappa_{s} / \kappa_{1}\right) \sin \left(\kappa_{1} x\right)+\cos \left(\kappa_{1} x\right)\right)$. At the point $x=h_{1}$, one gets

$$
\begin{aligned}
& y_{1}\left(h_{1}\right)=A\left(\frac{\kappa_{s}}{\kappa_{1}} \sin \left(\kappa_{1} h_{1}\right)+\cos \left(\kappa_{1} h_{1}\right)\right), \\
& y_{1}^{\prime}\left(h_{1}\right)=A \kappa_{1}\left(\frac{\kappa_{s}}{\kappa_{1}} \cos \left(\kappa_{1} h_{1}\right)-\sin \left(\kappa_{1} h_{1}\right)\right) .
\end{aligned}
$$

Substituting (71) into (48), one obtains

$$
\begin{aligned}
C_{2}= & \kappa_{1}^{2}\left(\frac{\kappa_{s}}{\kappa_{1}} \cos \left(\kappa_{1} h_{1}\right)-\sin \left(\kappa_{1} h_{1}\right)\right)^{2} A^{2} \\
& +\kappa_{2}^{2}\left(\frac{\kappa_{s}}{\kappa_{1}} \sin \left(\kappa_{1} h_{1}\right)+\cos \left(\kappa_{1} h_{1}\right)\right)^{2} A^{2} \\
& +\frac{1}{2} \beta_{2}\left(\frac{\kappa_{s}}{\kappa_{1}} \sin \left(\kappa_{1} h_{1}\right)+\cos \left(\kappa_{1} h_{1}\right)\right)^{4} A^{4} .
\end{aligned}
$$

If $k_{0}^{2} \varepsilon_{s}<\gamma^{2} \leqslant k_{0}^{2} \varepsilon_{1}$, then $\kappa_{1}^{2} \geqslant 0$ and $\kappa_{2}^{2} \geqslant 0$ and, therefore, $C_{2}>0$.

If $k_{0}^{2} \varepsilon_{1}<\gamma^{2} \leqslant k_{0}^{2} \varepsilon_{2}$, then $\kappa_{1}^{2}<0$ and $\kappa_{2}^{2} \geqslant 0$. As before, we denote $\kappa_{1}=i \widetilde{\kappa}_{1}$, where $\widetilde{\kappa}_{1}^{2}=\gamma^{2}-k_{0}^{2} \varepsilon_{1}>0$. Then

$$
\begin{aligned}
C_{2}= & \widetilde{\kappa}_{1}^{2}\left(\frac{\kappa_{s}}{\widetilde{\kappa}_{1}} \cosh \left(\widetilde{\kappa}_{1} h_{1}\right)+\sinh \left(\widetilde{\kappa}_{1} h_{1}\right)\right)^{2} A^{2} \\
& +\kappa_{2}^{2}\left(\frac{\kappa_{s}}{\widetilde{\kappa}_{1}} \sinh \left(\widetilde{\kappa}_{1} h_{1}\right)+\cosh \left(\widetilde{\kappa}_{1} h_{1}\right)\right)^{2} A^{2} \\
& +\frac{1}{2} \beta_{2}\left(\frac{\kappa_{s}}{\widetilde{\kappa}_{1}} \sinh \left(\kappa_{1} h_{1}\right)+\cosh \left(\widetilde{\kappa}_{1} h_{1}\right)\right)^{4} A^{4}
\end{aligned}
$$

$>0$.
If $\gamma^{2}>k_{0}^{2} \varepsilon_{2}$, then $\kappa_{1}^{2}<0$ and $\kappa_{2}^{2}<0$. As before, we denote $\kappa_{1}=i \widetilde{\kappa}_{1}$ and $\kappa_{2}=i \widetilde{\kappa}_{2}$, where $\widetilde{\kappa}_{1}^{2}=\gamma^{2}-k_{0}^{2} \varepsilon_{1}>0$ and $\widetilde{\kappa}_{2}^{2}=$ $\gamma^{2}-k_{0}^{2} \varepsilon_{2}>0$. Then

$$
\begin{aligned}
C_{2}= & \widetilde{\kappa}_{1}^{2}\left(\frac{\kappa_{s}}{\widetilde{\kappa}_{1}} \cosh \left(\widetilde{\kappa}_{1} h_{1}\right)+\sinh \left(\widetilde{\kappa}_{1} h_{1}\right)\right)^{2} A^{2} \\
& -\widetilde{\kappa}_{2}^{2}\left(\frac{\kappa_{s}}{\widetilde{\kappa}_{1}} \sinh \left(\widetilde{\kappa}_{1} h_{1}\right)+\cosh \left(\widetilde{\kappa}_{1} h_{1}\right)\right)^{2} A^{2} \\
& +\frac{1}{2} \beta_{2}\left(\frac{\kappa_{s}}{\widetilde{\kappa}_{1}} \sinh \left(\kappa_{1} h_{1}\right)+\cosh \left(\widetilde{\kappa}_{1} h_{1}\right)\right)^{4} A^{4} .
\end{aligned}
$$

Grouping terms in the last expression for $C_{2}$, one gets

$$
\begin{aligned}
& \frac{\kappa_{s}}{\widetilde{\kappa}_{1}} \cosh \left(\widetilde{\kappa}_{1} h_{1}\right)+\sinh \left(\widetilde{\kappa}_{1} h_{1}\right) \\
& =\frac{1}{2}\left(\frac{\kappa_{s}}{\widetilde{\kappa}_{1}}\left(e^{\widetilde{\kappa}_{1} h_{1}}+e^{-\widetilde{\kappa}_{1} h_{1}}\right)+\left(e^{\widetilde{\kappa}_{1} h_{1}}-e^{-\widetilde{\kappa}_{1} h_{1}}\right)\right) \\
& =\frac{1}{2}\left(a_{+} e^{\widetilde{\kappa}_{1} h_{1}}+a_{-} e^{-\widetilde{\kappa}_{1} h_{1}}\right), \\
& \frac{\kappa_{s}}{\widetilde{\kappa}_{1}} \sinh \left(\widetilde{\kappa}_{1} h_{1}\right)+\cosh \left(\widetilde{\kappa}_{1} h_{1}\right) \\
& =\frac{1}{2}\left(\frac{\kappa_{s}}{\widetilde{\kappa}_{1}}\left(e^{\widetilde{\kappa}_{1} h_{1}}-e^{-\widetilde{\kappa}_{1} h_{1}}\right)+\left(e^{\widetilde{\kappa}_{1} h_{1}}+e^{-\widetilde{\kappa}_{1} h_{1}}\right)\right) \\
& =\frac{1}{2}\left(a_{+} e^{\widetilde{\kappa}_{1} h_{1}}-a_{-} e^{-\widetilde{\kappa}_{1} h_{1}}\right),
\end{aligned}
$$

where $a_{+}:=\kappa_{s} / \widetilde{\kappa}_{1}+1$ and $a_{-}:=\kappa_{s} / \widetilde{\kappa}_{1}-1$.

Then

$$
\begin{aligned}
C_{2}= & \frac{1}{32} \beta_{2} A^{4} a_{+}^{4} e^{4 \widetilde{\kappa}_{1} h_{1}} \\
& +\frac{1}{4} A^{2}\left(k_{0}^{2} \varepsilon_{2}-k_{0}^{2} \varepsilon_{1}-\frac{1}{2} \beta_{2} a_{+} a_{-} A^{2}\right) a_{+}^{2} e^{2 \widetilde{\kappa}_{1} h_{1}} \\
& +\frac{1}{2} A^{2}\left(\widetilde{\kappa}_{1}^{2}+\widetilde{\kappa}_{2}^{2}+\frac{3}{8} \beta_{2} a_{+} a_{-} A^{2}\right) a_{+} a_{-} \\
& +\frac{1}{4} A^{2}\left(k_{0}^{2} \varepsilon_{2}-k_{0}^{2} \varepsilon_{1}-\frac{1}{2} \beta_{2} a_{+} a_{-} A^{2}\right) a_{-}^{2} e^{-2 \widetilde{\kappa}_{1} h_{1}} \\
& +\frac{1}{32} \beta_{2} A^{4} a_{-}^{4} e^{-4 \widetilde{\kappa}_{1} h_{1}} .
\end{aligned}
$$

Since $a_{+}>1$ and $a_{-}>0$ are bounded above, then it is clear that for sufficiently small $\beta_{2}>0$ the constant $C_{2}>0$ for all $\gamma^{2}>k_{0}^{2} \varepsilon_{2}$. It is also clear that for any fixed $\beta_{2}>0$ there exists $\gamma_{*}$ such that $C_{2}>0$ for all $\gamma>\gamma_{*}$.

Using the expansion $(1+x)^{\alpha}=1+O(x)$, where $|x|<$ 1 and $-1<\alpha<1$, for sufficiently large $\gamma$, one gets the asymptotics

$$
C_{2}=\beta_{2} A^{4}\left(\frac{1}{2}+O\left(\gamma^{-2}\right)\right) e^{4 h_{1} \gamma} .
$$

Since both integrands in (69) are positive, the estimate

$$
n_{2} T \leqslant \Phi_{2}\left(\gamma ; n_{2}, p\right) \leqslant\left(n_{2}+1\right) T
$$


takes place, where $n_{2} \geqslant 0$ and $T=\int_{-\infty}^{+\infty}\left(d s / w_{2}\right)$. Thus we need to evaluate $T$. For further analysis, we use the easy checked inequalities $1 /(a+b) \leqslant 1 / \sqrt{a^{2}+b^{2}} \leqslant \sqrt{2} /(a+b)$, where $a \geqslant 0$ and $b>0$. These inequalities give

$$
T^{*} \leqslant T \leqslant \sqrt{2} T^{*},
$$

where $T^{*}=\int_{-\infty}^{+\infty}\left(d s /\left(\left|s^{2}+\kappa_{2}^{2}\right|+\sqrt{2 \beta_{2} C_{2}}\right)\right)$. Integral $T^{*}$ is calculated explicitly. has

For $\gamma^{2}<k_{0}^{2} \varepsilon_{2}$, the value $\eta^{2}+\kappa_{2}^{2}>0$ and, therefore, one

$$
\begin{aligned}
T^{*} & =2 \int_{0}^{+\infty} \frac{d s}{\left|s^{2}+\kappa_{2}^{2}\right|+\sqrt{2 \beta_{2} C_{2}}} \\
& =2 \int_{0}^{+\infty} \frac{d s}{s^{2}+\kappa_{2}^{2}+\sqrt{2 \beta_{2} C_{2}}}=\frac{\pi}{\sqrt{\left|\kappa_{2}^{2}\right|+\sqrt{2 \beta_{2} C_{2}}}} .
\end{aligned}
$$

Let $\gamma^{2} \geqslant k_{0}^{2} \varepsilon_{2}$; then

$$
\begin{aligned}
T^{*}= & 2 \int_{0}^{+\infty} \frac{d s}{\left|s^{2}+\kappa_{2}^{2}\right|+\sqrt{2 \beta_{2} C_{2}}} \\
= & 2 \int_{0}^{\left|\kappa_{2}\right|} \frac{d s}{-s^{2}-\kappa_{2}^{2}+\sqrt{2 \beta_{2} C_{2}}} \\
& +2 \int_{\left|\kappa_{2}\right|}^{+\infty} \frac{d s}{s^{2}+\kappa_{2}^{2}+\sqrt{2 \beta_{2} C_{2}}} \\
= & 2 \int_{0}^{\left|\kappa_{2}\right|} \frac{d s}{\left|\kappa_{2}^{2}\right|+\sqrt{2 \beta_{2} C_{2}}-s^{2}} \\
& +2 \int_{\left|\kappa_{2}\right|}^{+\infty} \frac{d s}{s^{2}+\sqrt{2 \beta_{2} C_{2}}-\left|\kappa_{2}^{2}\right|}=2 I_{1}+2 I_{2} .
\end{aligned}
$$

Obviously, the denominator in $I_{1}$ is always positive.
Calculating $I_{1}$, one gets

$$
\begin{aligned}
I_{1} & =\int_{0}^{\left|\kappa_{2}\right|} \frac{d s}{\left|\kappa_{2}^{2}\right|+\sqrt{2 \beta_{2} C_{2}}-s^{2}} \\
& =\frac{-1}{2 \sqrt{\left|\kappa_{2}^{2}\right|+\sqrt{2 \beta_{2} C_{2}}}} \ln \frac{\sqrt{2 \beta_{2} C_{2}}}{\left(\left|\kappa_{2}\right|+\sqrt{\left|\kappa_{2}^{2}\right|+\sqrt{2 \beta_{2} C_{2}}}\right)^{2}} .
\end{aligned}
$$

Two cases are possible for $I_{2}$. If $\left|\kappa_{2}^{2}\right|-\sqrt{2 \beta_{2} C_{2}} \geqslant 0$, that is, $\gamma^{2} \geqslant k_{0}^{2} \varepsilon_{2}+\sqrt{2 \beta_{2} C_{2}}$, then

$$
\begin{aligned}
& I_{2}=\int_{\left|\kappa_{2}\right|}^{+\infty} \frac{d s}{s^{2}-\left(\left|\kappa_{2}^{2}\right|-\sqrt{2 \beta_{2} C_{2}}\right)} \\
& =-\frac{1}{2 \sqrt{\left|\kappa_{2}^{2}\right|-\sqrt{2 \beta_{2} C_{2}}}} \ln \frac{\sqrt{2 \beta_{2} C_{2}}}{\left(\left|\kappa_{2}\right|+\sqrt{\left|\kappa_{2}^{2}\right|-\sqrt{2 \beta_{2} C_{2}}}\right)^{2}} . \\
& \text { If }-\left|\kappa_{2}^{2}\right|+\sqrt{2 \beta_{2} C_{2}}>0 \text {, that is, } k_{0}^{2} \varepsilon_{2} \leqslant \gamma^{2}<k_{0}^{2} \varepsilon_{2}+\sqrt{2 \beta_{2} C_{2}},
\end{aligned}
$$
then

$$
\begin{aligned}
I_{2} & =\int_{\left|\kappa_{2}\right|}^{+\infty} \frac{d s}{s^{2}-\left|\kappa_{2}^{2}\right|+\sqrt{2 \beta_{2} C_{2}}} \\
& =\frac{1}{\sqrt{-\left|\kappa_{2}^{2}\right|+\sqrt{2 \beta_{2} C_{2}}}}\left(\frac{\pi}{2}\right. \\
& \left.-\arctan \frac{\left|\kappa_{2}\right|}{\sqrt{-\left|\kappa_{2}^{2}\right|+\sqrt{2 \beta_{2} C_{2}}}}\right) .
\end{aligned}
$$

Combining the found results, one arrives at the formula

$$
T^{*}= \begin{cases}\frac{\pi}{\theta}, & \gamma^{2}<k_{0}^{2} \varepsilon_{2} \\ -\frac{1}{\theta} \ln \frac{\sqrt{2 \beta_{2} C_{2}}}{\left(\left|\kappa_{2}\right|+\theta\right)^{2}}+\frac{2}{\theta_{1}}\left(\frac{\pi}{2}-\arctan \frac{\left|\kappa_{2}\right|}{\theta_{1}}\right), & k_{0}^{2} \varepsilon_{2} \leqslant \gamma^{2} \leqslant k_{0}^{2} \varepsilon_{2}+\sqrt{2 \beta_{2} C_{2}} \\ -\frac{1}{\theta} \ln \frac{\sqrt{2 \beta_{2} C_{2}}}{\left(\left|\kappa_{2}\right|+\theta\right)^{2}}-\frac{1}{\theta_{2}} \ln \frac{\sqrt{2 \beta_{2} C_{2}}}{\left(\left|\kappa_{2}\right|+\theta_{2}\right)^{2}}, & \gamma^{2} \geqslant k_{0}^{2} \varepsilon_{2}+\sqrt{2 \beta_{2} C_{2}}\end{cases}
$$

where $\theta=\sqrt{\left|\kappa_{2}^{2}\right|+\sqrt{2 \beta_{2} C_{2}}}, \theta_{1}=\sqrt{-\left|\kappa_{2}^{2}\right|+\sqrt{2 \beta_{2} C_{2}}}$, and $\theta_{2}=$ $\sqrt{\left|\kappa_{2}^{2}\right|-\sqrt{2 \beta_{2} C_{2}}}$.

Taking into account formula (77), it is clear that the inequality $\gamma^{2} \geqslant k_{0}^{2} \varepsilon_{2}+\sqrt{2 \beta_{2} C_{2}}$ with fixed $\beta_{2}$ holds only if $\gamma$ belongs to a finite interval.

Taking into account the fact that for large $\gamma$ the value $C_{2}$ is asymptotically equal to $(1 / 2) \beta_{2} A^{4} e^{4 h_{1} \gamma}$, one finds that the second formula in (85) must be used for sufficiently big $\gamma$.

Further, we need the following expansions: $\ln (1+x)=$ $x+O\left(x^{2}\right)$ and $\arctan (x)=x+O\left(x^{3}\right)$, which are valid for $|x|<1$. Using these expansions for the second line in (85), one finds

$$
\begin{aligned}
T^{*} & =-\frac{1}{\sqrt[4]{2 \beta_{2} C_{2}}}\left(1+\frac{\left|\kappa_{2}^{2}\right|}{\sqrt{2 \beta_{2} C_{2}}}\right)^{-1 / 2} \\
& \left(\ln \sqrt{2 \beta_{2} C_{2}}-2 \ln \left(\left|\kappa_{2}\right|+\theta\right)\right) \\
& +\frac{2}{\sqrt[4]{2 \beta_{2} C_{2}}}\left(1-\frac{\left|\kappa_{2}^{2}\right|}{\sqrt{2 \beta_{2} C_{2}}}\right)^{-1 / 2}
\end{aligned}
$$




$$
\begin{aligned}
& \cdot\left(\frac{\pi}{2}-\frac{\left|\kappa_{2}\right|}{\theta_{1}}+O\left(\left(\frac{\left|\kappa_{2}\right|}{\theta_{1}}\right)^{2}\right)\right) \\
& =\frac{1}{\sqrt[4]{2 \beta_{2} C_{2}}}\left(\pi+O\left(\frac{\left|\kappa_{2}^{2}\right|}{\sqrt[4]{2 \beta_{2} C_{2}}}\right)\right) .
\end{aligned}
$$

Taking into account the fact that

$$
\begin{aligned}
\left(2 \beta_{2} C_{2}\right)^{-1 / 4} & =\left(2 \beta_{2} \cdot \frac{1}{2} \beta_{2} A^{4}\left(1+O\left(\gamma^{-2}\right)\right) e^{4 h_{1} \gamma}\right)^{-1 / 4} \\
& =\frac{e^{-h_{1} \gamma}}{A \sqrt{\beta_{2}}}\left(1+O\left(\gamma^{-2}\right)\right)
\end{aligned}
$$

one finally obtains

$$
T^{*}=\frac{\pi e^{-h_{1} \gamma}}{A \sqrt{\beta_{2}}}\left(1+O\left(\gamma^{-2}\right)\right)
$$

for sufficiently large $\gamma$.

It follows from (88) that $\lim _{\gamma^{2} \rightarrow \infty} T^{*}=0$. This means that for any $h_{1}, h_{2}>0$ there is an integer $n^{*} \geqslant 0$ such that, for any integer $n_{2} \geqslant n^{*}$, the DE (69) has at least one solution. This implies the existence of infinitely many $\widehat{\gamma}_{i}$ with an accumulation point at infinity.

Passing to the limit $\beta_{2} \rightarrow 0$ in the first line of (85), one obtains either (20) or (19) depending on $k_{0}^{2} \varepsilon_{s}<\gamma^{2}<k_{0}^{2} \varepsilon_{1}$ or $k_{0}^{2} \varepsilon_{1}<\gamma^{2}<k_{0}^{2} \varepsilon_{2}$. This implies item (2) of the theorem.

Multiplying (8) by $y_{2}$ and integrating over $\left[h_{1}, h_{1}+h_{2}\right]$, one gets the following for $\gamma^{2}>k_{0}^{2} \varepsilon_{2}$ :

$$
\int_{h_{1}}^{h_{1}+h_{2}} y_{2} y_{2}^{\prime \prime} d x=-\kappa_{2}^{2} \int_{h_{1}}^{h_{1}+h_{2}} y_{2}^{2} d x-\beta_{2} \int_{h_{1}}^{h_{1}+h_{2}} y_{2}^{4} d x .
$$

This gives

$$
\begin{aligned}
& \left.y_{2} y_{2}^{\prime}\right|_{h_{1}} ^{h_{1}+h_{2}}-\int_{h_{1}}^{h_{1}+h_{2}} y_{2}^{\prime 2} d x \\
& =-\kappa_{2}^{2} \int_{h_{1}}^{h_{1}+h_{2}} y_{2}^{2} d x-\beta_{2} \int_{h_{1}}^{h_{1}+h_{2}} y_{2}^{4} d x .
\end{aligned}
$$

Taking into account the fact that

$$
\begin{aligned}
& y_{2}\left(h_{1}\right) y_{2}^{\prime}\left(h_{1}\right) \\
&= \frac{A^{2}}{\widetilde{\kappa}_{1}}\left(\kappa_{s} \sinh \left(\widetilde{\kappa}_{1} h_{1}\right)+\widetilde{\kappa}_{1} \cosh \left(\widetilde{\kappa}_{1} h_{1}\right)\right) \\
& \cdot\left(\kappa_{s} \cosh \left(\widetilde{\kappa}_{1} h_{1}\right)+\widetilde{\kappa}_{1} \sinh \left(\widetilde{\kappa}_{1} h_{1}\right)\right)>0,
\end{aligned}
$$

where $\tilde{\kappa}_{1} h_{1}>0$ and $y_{2}\left(h_{1}+h_{2}\right)=0$, formula (90) results in

$$
\begin{aligned}
& \beta_{2} \int_{h_{1}}^{h_{1}+h_{2}} y_{2}^{4} d x \\
& \quad=\frac{A^{2}}{\widetilde{\kappa}_{1}}\left(\kappa_{s} \sinh \left(\widetilde{\kappa}_{1} h_{1}\right)+\widetilde{\kappa}_{1} \cosh \left(\widetilde{\kappa}_{1} h_{1}\right)\right) \\
& \cdot\left(\kappa_{s} \cosh \left(\widetilde{\kappa}_{1} h_{1}\right)+\widetilde{\kappa}_{1} \sinh \left(\widetilde{\kappa}_{1} h_{1}\right)\right)+\int_{h_{1}}^{h_{1}+h_{2}} y_{2}^{\prime 2} d x \\
& +\left(\gamma^{2}-k_{0}^{2} \varepsilon_{2}\right) \int_{h_{1}}^{h_{1}+h_{2}} y_{2}^{2} d x .
\end{aligned}
$$

If $\gamma \rightarrow \infty$, then, obviously, the right-hand side of this equality tends to $\infty$ and so does the left-hand side. This implies that $\lim _{\gamma^{2} \rightarrow \infty} \max _{x \in\left[h_{1}, h_{1}+h_{2}\right]}\left|y_{2}(x ; \gamma)\right|=\infty$.

For $\max _{x \in\left[h_{1}, h_{1}+h_{2}\right]}\left|y_{2}(x ; \gamma)\right|$ using (48), one can derive an exact formula provided that $y_{2}(x ; \gamma)$ has at least two zeros inside the segment.

In order to derive the asymptotic estimates for $\widehat{\gamma}_{i}$, let us get back to formula (88). It follows from (78) and (79) that

$$
\begin{aligned}
n_{2} T^{*} & \leqslant n_{2} T \leqslant \Phi_{2}\left(\gamma ; n_{2}, p\right) \leqslant\left(n_{2}+1\right) T \\
& \leqslant \sqrt{2}\left(n_{2}+1\right) T^{*} .
\end{aligned}
$$

Since $n_{2} T^{*}$ bounds $h_{2}$ from below and $\sqrt{2}\left(n_{2}+1\right) T^{*}$ bounds $h_{2}$ from above, then, solving the equations $h_{2}=$ $n_{2}\left(\pi e^{-h_{1} \gamma} / A \sqrt{\beta_{2}}\right)$ and $h_{2}=\sqrt{2}\left(n_{2}+1\right)\left(\pi e^{-h_{1} \gamma} / A \sqrt{\beta_{2}}\right)$, with respect to $\gamma$, one finds (70).

5.2. Case $\beta_{1} \neq 0$ and $\beta_{2}=0$. If $\beta_{2}=0$ (or $\alpha_{2}=0$ ), solutions to (8) are found elementarily. This allows one to explicitly compute the quantity $p$, which is

$$
p=\kappa_{2} \cot \left(\kappa_{2} h_{2}\right) \text {. }
$$

This expression (due to its analytical dependence on $\gamma$ ) can be used for $\kappa_{2}^{2} \geqslant 0$ as well as for $\kappa_{2}^{2} \leqslant 0$. Note that $p$ is a real value for all $\gamma^{2}>k_{0}^{2} \varepsilon_{s}$. Indeed, for $\gamma^{2}>k_{0}^{2} \varepsilon_{1}$, one has $\kappa_{2}=i \widetilde{\kappa}_{2}$, where $\widetilde{\kappa}_{2}^{2}=\gamma^{2}-k_{0}^{2} \varepsilon_{2}$ and then $\cos (i x)=\cosh x$ and $\sin (i x)=i \sinh x$; then

$$
\begin{aligned}
p & =\kappa_{2} \frac{\cos \left(\kappa_{2} h_{2}\right)}{\sin \left(\kappa_{2} h_{2}\right)}=i \widetilde{\kappa}_{2} \frac{\cosh \left(\widetilde{\kappa}_{2} h_{2}\right)}{i \sinh \left(\widetilde{\kappa}_{2} h_{2}\right)} \\
& =\widetilde{\kappa}_{2} \frac{\cosh \left(\widetilde{\kappa}_{2} h_{2}\right)}{\sinh \left(\widetilde{\kappa}_{2} h_{2}\right)} .
\end{aligned}
$$

It is clear that the substitution of the explicit expression for $p$ into the second equation of system (58) leads to the identity. Thus, in this case, system (58) reduces to the only equation

$$
\Phi_{1}\left(\gamma ; n_{1}, p\right)=h_{1},
$$

where $\Phi_{1}$ and $p$ are given by (46) and (94), respectively; $n_{1}=$ $0,1,2, \ldots$.

The solvability of the problem $P\left(\beta_{1}, 0\right)$ is established by the following. 
Theorem 3. For $\beta_{2}=0$, any $\beta_{1}>0$, and any fixed $A \neq 0$, the problem $P\left(\beta_{1}, 0\right)$ has an infinite number of PCs $\widehat{\gamma}_{i}(i=1,2, \ldots)$ with the following properties:

(1) If $\widehat{\gamma}_{i}$ is the solution to $P\left(\beta_{1}, 0\right)$, then $\widehat{\gamma}_{i}^{2} \in\left(k_{0}^{2} \varepsilon_{s},+\infty\right)$ and $\lim _{i \rightarrow \infty} \widehat{\gamma}_{i}^{2}=\infty$.

(2) If the linear problem $P(0,0)$ has q solutions $\tilde{\gamma}_{1}<\widetilde{\gamma}_{2}<$ $\cdots<\tilde{\gamma}_{q}$, then there exists a constant $\beta_{0}>0$ such that, for any $\beta_{1}=\beta_{1}^{\prime}<\beta_{0}$, it is true that $\widehat{\gamma}_{i}^{2} \in\left(k_{0}^{2} \varepsilon_{s}, k_{0}^{2} \varepsilon_{2}\right)$ and $\lim _{\beta_{1}^{\prime} \rightarrow 0} \widehat{\gamma}_{i}=\widetilde{\gamma}_{i}(i=\overline{1, q})$, where $\widehat{\gamma}_{1}, \widehat{\gamma}_{2}, \ldots, \widehat{\gamma}_{q}$ are first $q$ solutions to $P\left(\beta_{1}^{\prime}, 0\right)$.

(3) If $s>q$, then $\lim _{\beta_{1}^{\prime} \rightarrow+0} \widehat{\gamma}_{s}=+\infty$.

(4) If $\widehat{\gamma}_{i} \rightarrow \infty$, then $\max _{x \in\left(0, h_{1}\right)}\left|y_{2}\left(x, \widehat{\gamma}_{i}\right)\right| \rightarrow \infty$.

(5) For big $\hat{\gamma}$ and arbitrary small $\Delta>0$, the asymptotic two-sided inequality

$$
(1-\Delta) \gamma_{\bullet}(m) \leqslant \widehat{\gamma}(m) \leqslant(1+\Delta) \gamma_{\bullet}(\sqrt{2}(m+1))
$$

is valid, where $\gamma_{0}^{2}(m)=\varepsilon_{1}+\left[f^{-1}(h / 4 m)\right]^{2}$ and $f^{-1}$ is the inversion of $f(t)=t^{-1} \ln t$.

Proof. The constant $C_{1}$ in (96) is defined explicitly by (32).
Using (16) at the point $x=h_{1}$, one obtains

$$
\begin{aligned}
& y_{2}\left(h_{1}\right)=-B \sin \left(\kappa_{2} h_{2}\right), \\
& y_{2}^{\prime}\left(h_{1}\right)=B \kappa_{2} \cos \left(\kappa_{2} h_{2}\right) .
\end{aligned}
$$

Substituting these values into (31) and using (32), one obtains the biquadratic equation

$$
\begin{gathered}
B^{4}+2 \frac{\kappa_{1}^{2} \sin ^{2}\left(\kappa_{2} h_{2}\right)+\kappa_{2}^{2} \cos ^{2}\left(\kappa_{2} h_{2}\right)}{\beta_{1} \sin ^{4}\left(\kappa_{2} h_{2}\right)} B^{2} \\
-\frac{2 k_{0}^{2}\left(\varepsilon_{1}-\varepsilon_{s}\right)+\beta_{1} A^{2}}{\beta_{1} \sin ^{4}\left(\kappa_{2} h_{2}\right)} A^{2}=0 .
\end{gathered}
$$

Using the same estimations as in the proof of Theorem 2 and evaluating the integral contained in (96), one finds

$$
\begin{aligned}
n_{1} T^{*} & \leqslant n_{1} T \leqslant \Phi_{1}\left(\gamma ; n_{1}, p\right) \leqslant\left(n_{1}+1\right) T \\
& \leqslant \sqrt{2}\left(n_{1}+1\right) T^{*}
\end{aligned}
$$

where $T^{*}=\int_{-\infty}^{+\infty}\left(d \eta /\left(\left|\eta^{2}+\kappa_{1}^{2}\right|+\sqrt{2 \beta_{1} C_{1}}\right)\right)$.

Integral $T^{*}$ is computed in the same way as in the proof of Theorem 2; the result has the form

$$
T^{*}= \begin{cases}\frac{\pi}{\theta}, & \gamma^{2}<k_{0}^{2} \varepsilon_{1}, \\ -\frac{1}{\theta} \ln \frac{\sqrt{2 \beta_{1} C_{1}}}{\left(\left|\kappa_{1}\right|+\theta\right)^{2}}+\frac{2}{\theta_{1}}\left(\frac{\pi}{2}-\arctan \frac{\left|\kappa_{1}\right|}{\theta_{1}}\right), & k_{0}^{2} \varepsilon_{1} \leqslant \gamma^{2} \leqslant k_{0}^{2} \varepsilon_{1}+\sqrt{2 \beta_{1} C_{1}}, \\ -\frac{1}{\theta} \ln \frac{\sqrt{2 \beta_{1} C_{1}}}{\left(\left|\kappa_{1}\right|+\theta\right)^{2}}-\frac{1}{\theta_{2}} \ln \frac{\sqrt{2 \beta_{1} C_{1}}}{\left(\left|\kappa_{1}\right|+\theta_{2}\right)^{2}}, & \gamma^{2} \geqslant k_{0}^{2} \varepsilon_{1}+\sqrt{2 \beta_{1} C_{1}},\end{cases}
$$

where $\theta=\sqrt{\left|\kappa_{1}^{2}\right|+\sqrt{2 \beta_{1} C_{1}}}, \theta_{1}=\sqrt{-\left|\kappa_{1}^{2}\right|+\sqrt{2 \beta_{1} C_{1}}}$, and $\theta_{2}=$ $\sqrt{\left|\kappa_{1}^{2}\right|-\sqrt{2 \beta_{1} C_{1}}}$.

In order to derive the asymptotic estimates for the eigenvalues $\widehat{\gamma}_{i}$, let us get back to formula (101). Since $C_{1}$ is a (fixed) constant [it does not depend on $\gamma$; see formula (32)], the third line in (101) corresponds to sufficiently big $\gamma$. Thus, for sufficiently big $\gamma$, the third line of (101) gives the asymptotics

$$
T^{*}=4 \frac{\ln \gamma}{\gamma}+O\left(\gamma^{-1}\right) .
$$

It follows from (102) that $\lim _{\gamma^{2} \rightarrow \infty} T^{*}=0$. This implies that for any prescribed $h_{1}, h_{2}>0$ there is an integer $n^{*} \geqslant 0$ such that (96) has at least one solution for any integer $n_{1} \geqslant n^{*}$. Thus, there exists an infinite number of positive eigenvalues $\widehat{\gamma}_{i}$ with accumulation point at infinity.

Passing to the limit $\beta_{1} \rightarrow+0$ in the first line of (101) gives either (20) or (19) depending on $k_{0}^{2} \varepsilon_{s}<\gamma^{2}<k_{0}^{2} \varepsilon_{1}$ or $k_{0}^{2} \varepsilon_{1}<$ $\gamma^{2}<k_{0}^{2} \varepsilon_{2}$. This implies item (2) of the theorem.

Item (3) of the theorem results from the behaviour of the second and third lines in (101) as $\beta_{1} \rightarrow+0$.
Multiplying (7) by $y_{1}$ and integrating over $\left[0, h_{1}\right]$, one obtains

$$
\begin{gathered}
y_{2}\left(h_{1}\right) y_{2}^{\prime}\left(h_{1}\right)-\kappa_{s} A^{2}+\int_{0}^{h_{1}} y_{1}^{\prime 2} d x \\
=\kappa_{1}^{2} \int_{0}^{h_{1}} y_{1}^{2} d x+\beta_{1} \int_{0}^{h_{1}} y_{1}^{4} d x .
\end{gathered}
$$

It follows from (103) that $\int_{0}^{h_{1}} y_{1}^{4} d x \rightarrow \infty$ as $\gamma \rightarrow \infty$. This implies item (4) of the theorem.

The asymptotic estimates in the theorem result from formula (102).

\section{Numerical Results}

Figures 2-13 show results of numerical experiments. The following parameters are used: $A=1[\mathbf{E}]$ and $\varepsilon_{s}=1 ; \varepsilon_{1}=2$ and $\varepsilon_{2}=2.5$ (Figures 2-9); $\varepsilon_{1}=5$ and $\varepsilon_{2}=10$ (Figures 10 and 11); $\varepsilon_{1}=5$ and $\varepsilon_{2}=5.01$ (Figures 12 and 13); other parameters are specified in the captions; $\omega=2 \pi f / c$, where $c$ is the speed of light. 


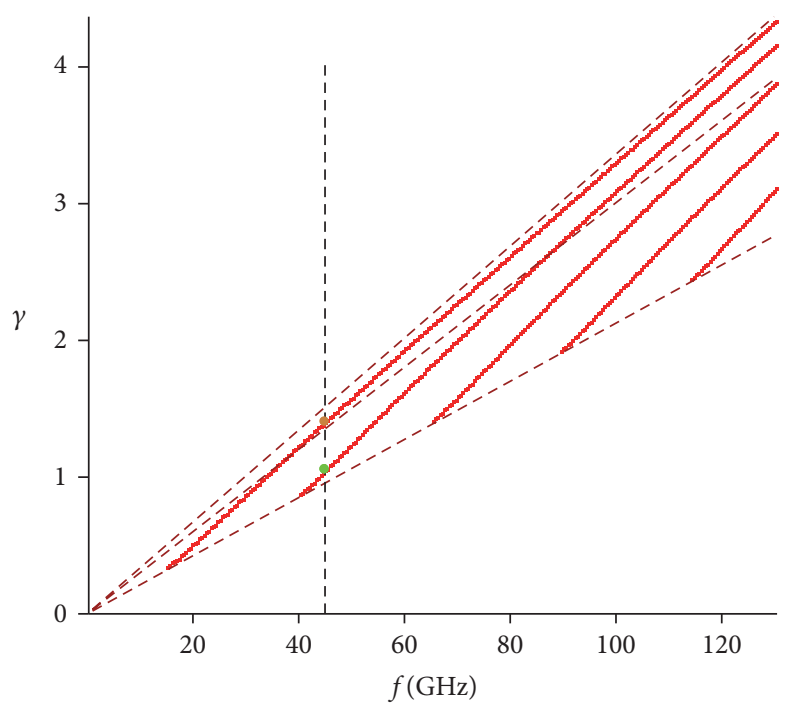

FiguRE 2: DCs for the linear case: $h_{1}=1$ and $h_{2}=4[\mathrm{~mm}]$; vertical dashed line $f=45[\mathrm{GHz}] ; \tilde{\gamma}_{1} \approx 1.0170$ (green dot) and $\tilde{\gamma}_{2} \approx 1.3709$ (brown dot).

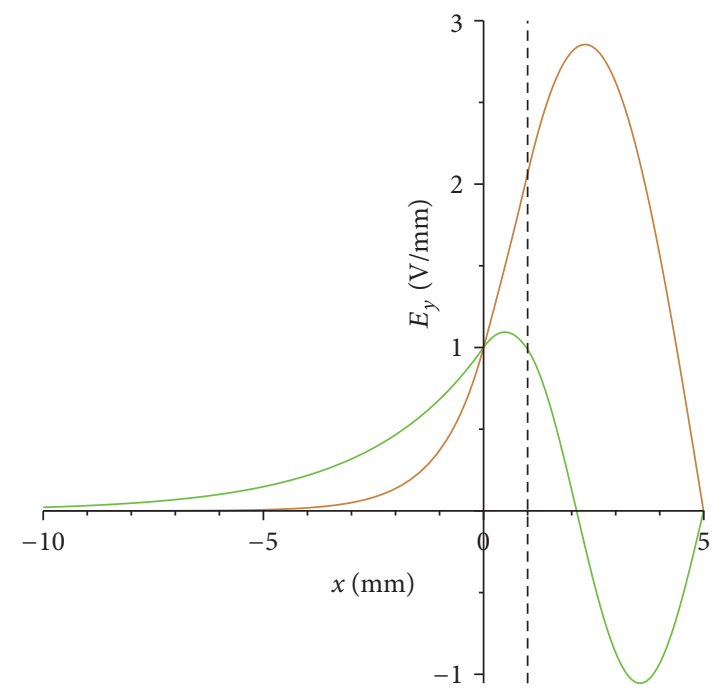

Figure 3: Eigenmodes $E_{y}(x)$ for the PCs marked in Figure 2: $\tilde{\gamma}_{1} \approx 1.0170$ (green) and $\widetilde{\gamma}_{2} \approx 1.3709$ (brown).

In Figures 2, 4, 6, 8, 10, and 12(a), the dispersion curves (DCs) are plotted ( $\gamma$ versus $f$ ). The DCs for the linear and nonlinear cases are given in red and blue colours, respectively. Dashed lines in Figure 2 correspond to the boundaries for $\gamma$. Points of intersections of the vertical dashed line with DCs are PCs.

Dashed lines in Figures 3, 5, 7, and 9 correspond to the boundary between the layers.

Figures 2 and 3 correspond to the linear problem $\left(\alpha_{1}=\right.$ $\alpha_{2}=0$ ). For chosen frequency, there are 2 PCs; eigenmodes for them are shown in Figure 3.

The dependence $\gamma$ versus $A$, where $A=E_{y}(0)$, is shown in Figures 2, 4, 6, 8, 10, and 12(b). The linear and nonlinear cases are given in red and blue colours, respectively. Points of intersections of the vertical dashed line (corresponding to
$A=1$ ) with the curves are PCs. Obviously, in the linear case, the PCs do not depend on $A$ and therefore linear solutions are horizontal (red) lines.

There are two types of PCs for the nonlinear case. The first type corresponds to the PCs, which are close to the corresponding linear solutions (in the linear limit, they reduce to the linear solutions; see item (2) in Theorems 2 and 3 . The second type corresponds to the PCs, which present a novel guiding regime (they do not reduce to any linear solutions in the linear limit; see item (3) in Theorem 3). In the latter case, we call them "purely nonlinear" PCs; see also [23-25]. In Figures 4, 6, and 8, the PC $\widehat{\gamma}_{1}$ marked with a blue dot corresponds to the first type; the PCs $\widehat{\gamma}_{2}$ and $\widehat{\gamma}_{3}$ marked with green and brown dots, respectively, correspond to the second type. 


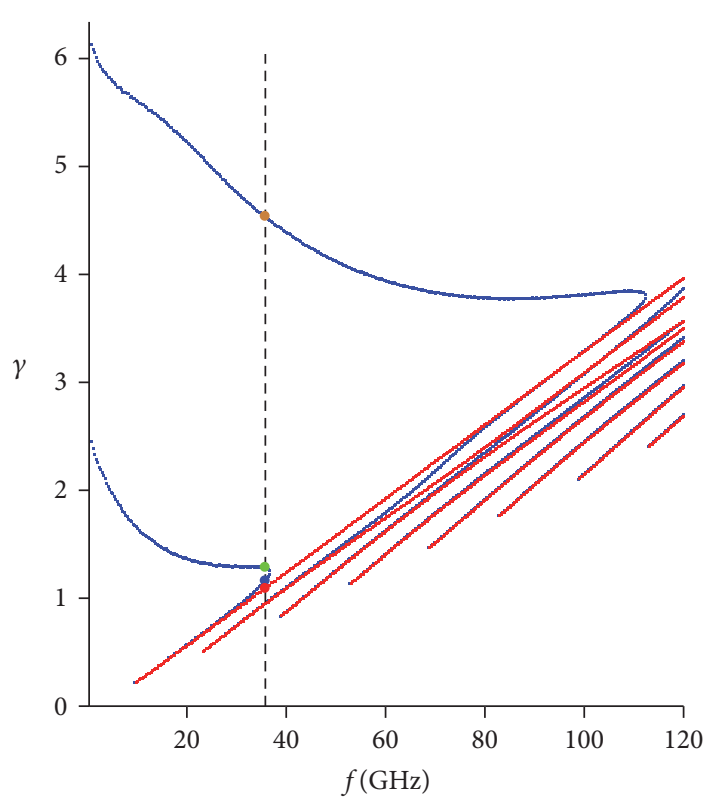

(a)

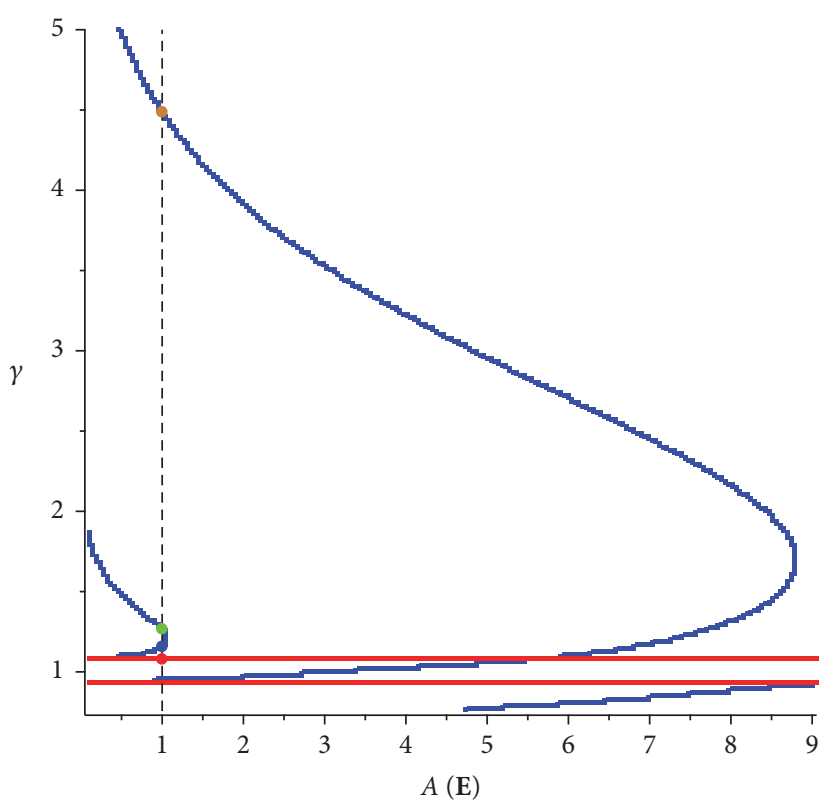

(b)

Figure 4: (a) DCs for nonlinear (blue) and linear (red) cases: $\alpha_{1}=0.02\left[\mathbf{E}^{-2}\right], \alpha_{2}=0, h_{1}=5, h_{2}=4[\mathrm{~mm}]$, and $E_{y}(0)=1[\mathbf{E}]$; vertical dashed line $f=36.1[\mathrm{GHz}]$; nonlinear PCs: $\widehat{\gamma}_{1} \approx 1.1509$ (blue dot), $\widehat{\gamma}_{2} \approx 1.2701$ (green dot), and $\widehat{\gamma}_{3} \approx 4.5003$ (brown dot), respectively; linear PC: $\widetilde{\gamma}_{2} \approx 1.0860$ (red dot). (b) The dependence $\gamma$ versus $A$ for nonlinear (blue) and linear (red) cases: $\alpha_{1}=0.02\left[\mathbf{E}^{-2}\right], \alpha_{2}=0, h_{1}=5, h_{2}=4[\mathrm{~mm}]$, and $f=36.1[\mathrm{GHz}]$; vertical dashed line $E_{y}(0)=1[\mathrm{E}]$; nonlinear PCs: $\widehat{\gamma}_{1} \approx 1.1509$ (blue dot), $\widehat{\gamma}_{2} \approx 1.2701$ (green dot), and $\widehat{\gamma}_{3} \approx 4.5003$ (brown dot), respectively; linear PC: $\widetilde{\gamma}_{2} \approx 1.0860$ (red dot).
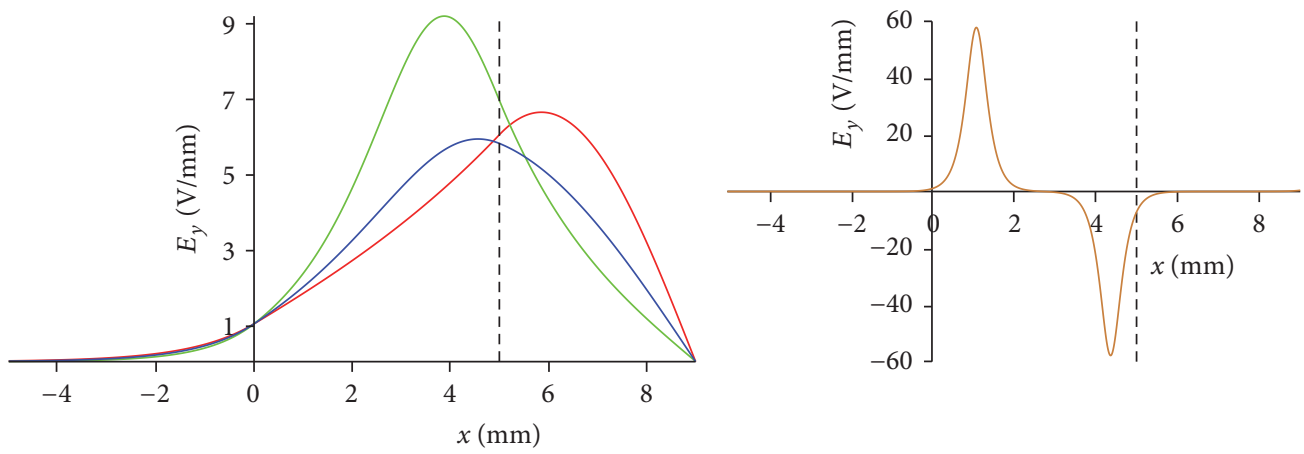

FIGURE 5: Eigenmodes $E_{y}(x)$ for the PCs marked in Figure 4: $\widetilde{\gamma}_{2} \approx 1.0860$ (red, linear case), $\widehat{\gamma}_{1} \approx 1.1509$ (blue), $\widehat{\gamma}_{2} \approx 1.2701$ (green), and $\widehat{\gamma}_{3} \approx 4.5003$ (brown).

In Figures 6 and 8, for any frequency, there are infinitely many PCs in the nonlinear cases (few of them are shown and marked in the figures) and only 2 PCs in the linear case.

DCs for the linear cases (red DCs) presented in Figures 6 and 8 coincide with the DCs for the linear case presented in Figure 2.

Figures 5, 7, and 9 allow one to compare linear and nonlinear modes. It can be seen from these pictures that, for a "linear" PC and for a "nonlinear" one (which reduces to the "linear" one in the linear limit), eigenmodes are also close. Obviously, for such a nonlinear PC, perturbation methods can be applied. However, two other nonlinear eigenmodes (shown in green and brown colours) are not close to any linear solution and they do not reduce to linear solutions in the linear limit; these very eigenmodes we call purely nonlinear.

In Figures 8-13, the nonlinear case for different sets of $\alpha_{1}, \alpha_{2}$ is shown, where both coefficients $\alpha_{1}$ and $\alpha_{2}$ are nonzero. In this case, we did not prove the existence of infinitely many PCs; however, these figures are similar to corresponding figures, where $\alpha_{1}$ or $\alpha_{2}$ equals zero.

\section{Conclusion}

The paper focuses on the problem of wave propagation in a plane layered dielectric waveguide filled with Kerr medium. The existence of guided modes that have linear counterparts 


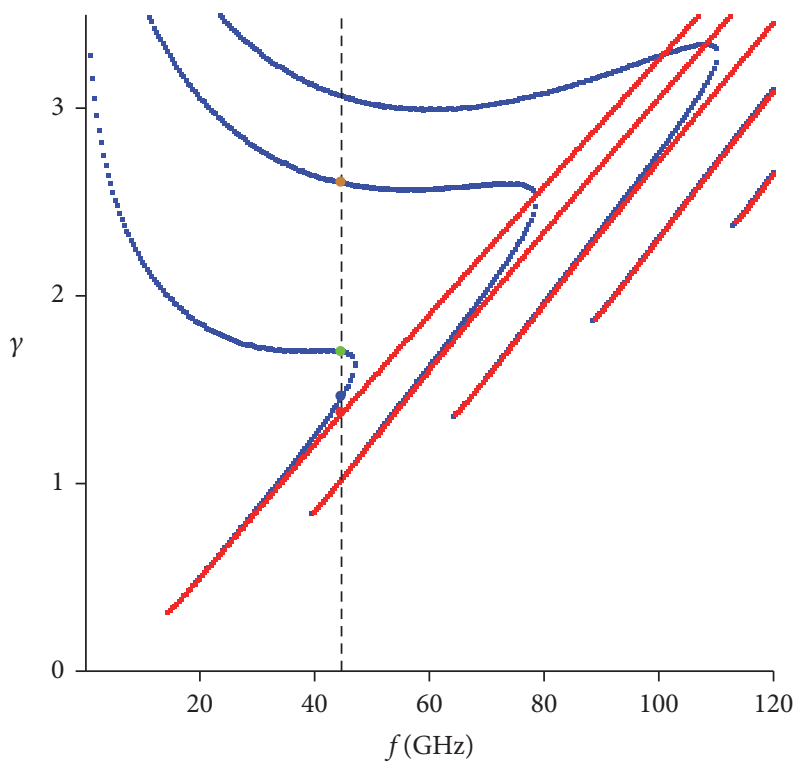

(a)

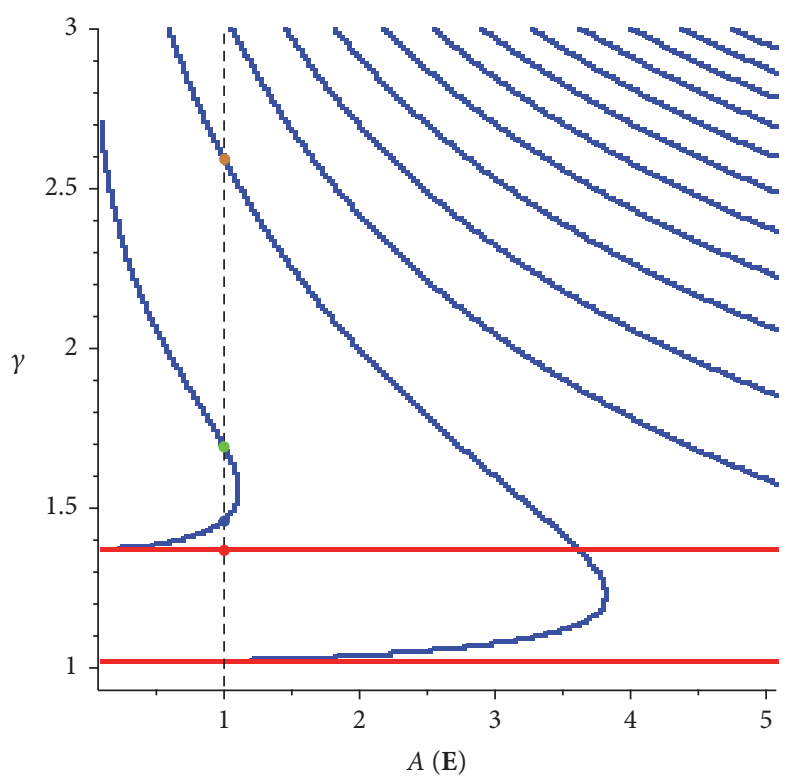

(b)

Figure 6: (a) DCs for nonlinear (blue) and linear (red) cases: $\alpha_{1}=0, \alpha_{2}=0.03\left[\mathbf{E}^{-2}\right], h_{1}=1[\mathrm{~mm}], h_{2}=4[\mathrm{~mm}]$, and $E_{y}(0)=1[\mathrm{E}]$; vertical dashed line $f=45$ [GHz]; nonlinear PCs: $\widehat{\gamma}_{1} \approx 1.4625$ (blue dot), $\widehat{\gamma}_{2} \approx 1.7012$ (green dot), and $\widehat{\gamma}_{3} \approx 2.6038$ (brown dot), respectively. The linear case coincides with one presented in Figure 2. (b) The dependence $\gamma$ versus $A$ for nonlinear (blue) and linear (red) cases: $\alpha_{1}=0$, $\alpha_{2}=0.03\left[\mathbf{E}^{-2}\right], h_{1}=1[\mathrm{~mm}], h_{2}=4[\mathrm{~mm}]$, and $f=45[\mathrm{GHz}]$; vertical dashed line $E_{y}(0)=1[\mathbf{E}]$; nonlinear PCs: $\widehat{\gamma}_{1} \approx 1.4625(\mathrm{blue}$ dot), $\widehat{\gamma}_{2} \approx 1.7012$ (green dot), and $\widehat{\gamma}_{3} \approx 2.6038$ (brown dot), respectively; linear PC: $\widetilde{\gamma}_{2} \approx 1.3709$ (red dot).

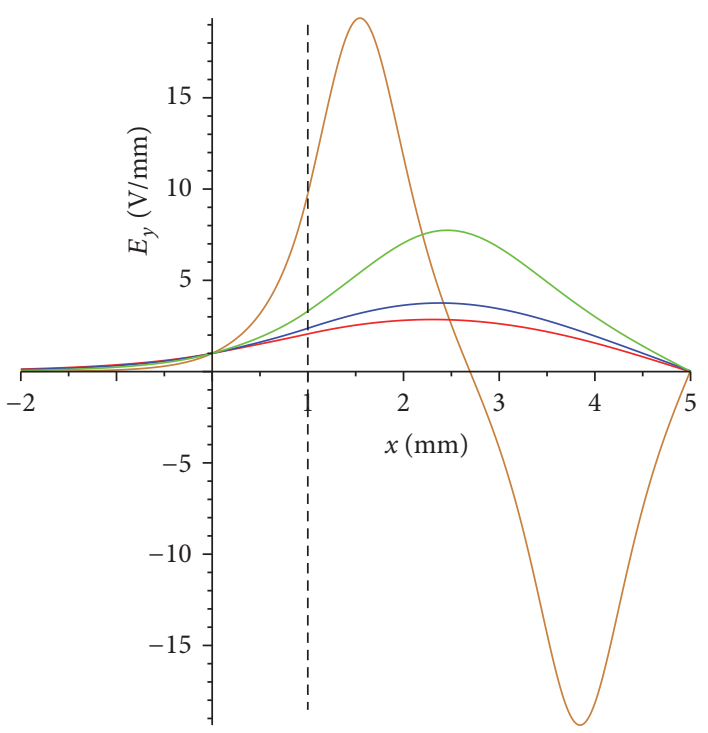

Figure 7: Eigenmodes $E_{y}(x): \widetilde{\gamma}_{2} \approx 1.3709$ (red, linear case), $\widehat{\gamma}_{1} \approx 1.4625$ (blue), $\widehat{\gamma}_{2} \approx 1.7012$ (green), and $\widehat{\gamma}_{3} \approx 2.6038$ (brown).

and guided modes that do not have linear counterparts is proven. The latter waves correspond to a novel guided regime. Since the Kerr nonlinearity is widely studied in nonlinear optics (see, e.g., [7-14]), the results found here can be interesting and important from both theoretical and applied points of view.

It is worth noting that similar results have been found for some other cases. Indeed, it was proven lately that, even in a simpler case of a one-layer waveguide, the Kerr nonlinearity results in the existence of novel guided regimes as well [2325]. Moreover, similar results have recently been found in the case of polynomial nonlinearity [39]. Thus, the existence of infinitely many nonperturbative PCs is a general feature of polynomial (nonlinear) permittivities with positive terms.

One of the most known peculiarities of nonlinear guided waves is power-dependent PCs; this is clearly shown in Figures 2, 4, 6, 8, 10, and 12(b). It is interesting that varying the value $A$, one strongly affects the corresponding $\mathrm{PC}$ and 


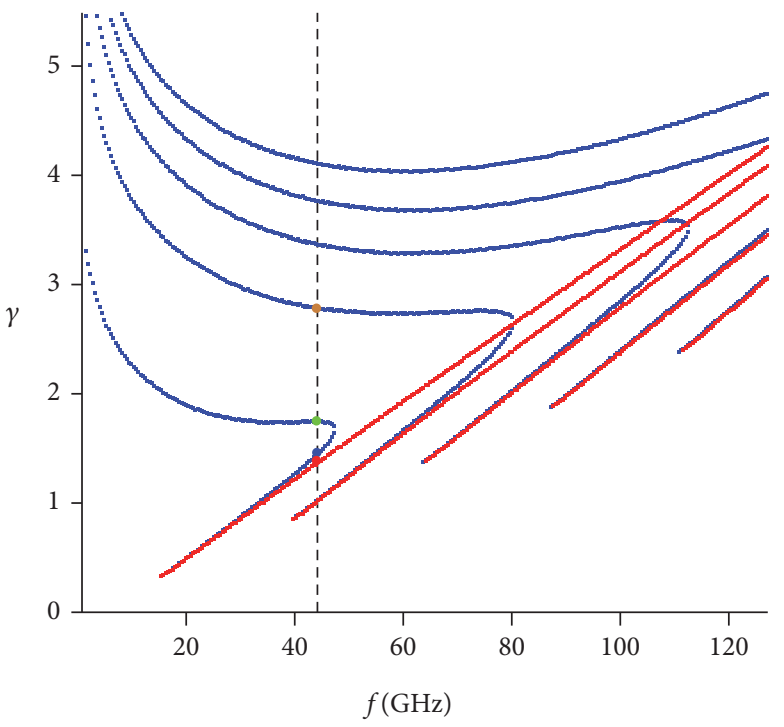

(a)

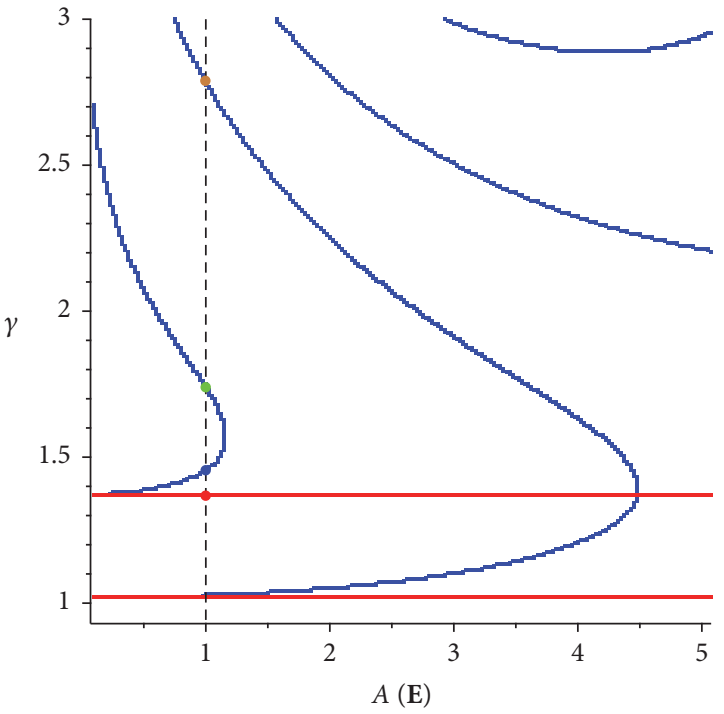

(b)

Figure 8: (a) DCs for nonlinear (blue) and linear (red) cases: $\alpha_{1}=0.02, \alpha_{2}=0.03\left[\mathbf{E}^{-2}\right], h_{1}=1, h_{2}=4[\mathrm{~mm}]$, and $E_{y}(0)=1[\mathrm{E}]$; black dashed line $f=45[\mathrm{GHz}]$; nonlinear PCs: $\widehat{\gamma}_{1} \approx 1.4548$ (blue dot), $\widehat{\gamma}_{2} \approx 1.7476$ (green dot), and $\widehat{\gamma}_{3} \approx 2.7896$ (brown dot), respectively. The linear case coincides with one presented in Figure 2. (b) The dependence $\gamma$ versus $A$ for nonlinear (blue) and linear (red) cases: $\alpha_{1}=0.02$, $\alpha_{2}=0.03\left[\mathbf{E}^{-2}\right], h_{1}=1, h_{2}=4[\mathrm{~mm}]$, and $f=45[\mathrm{GHz}]$; black dashed line $E_{y}(0)=1[\mathrm{E}] ;$ nonlinear PCs: $\widehat{\gamma}_{1} \approx 1.4548$ (blue dot), $\widehat{\gamma}_{2} \approx 1.7476$ (green dot), and $\widehat{\gamma}_{3} \approx 2.7896$ (brown dot), respectively; linear PC: $\widetilde{\gamma}_{2} \approx 1.3709$ (red dot).

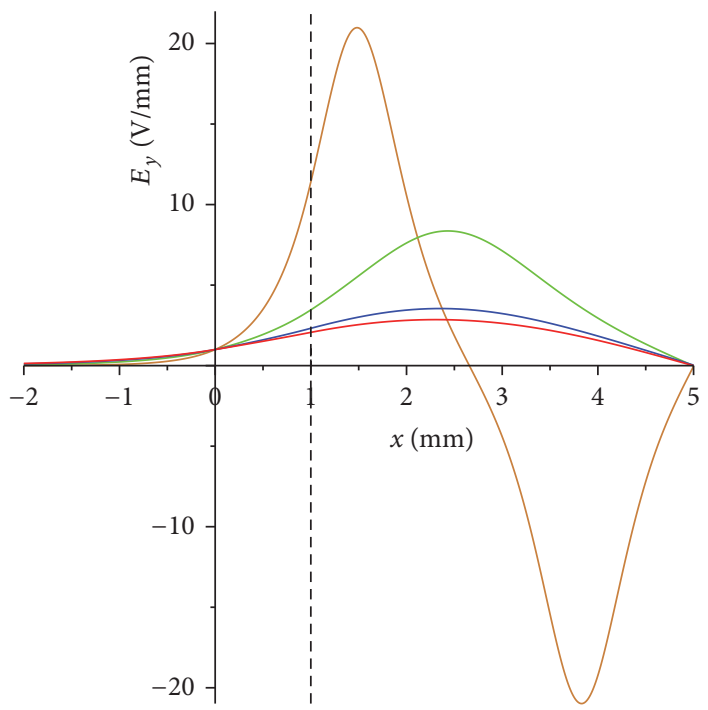

FIGURE 9: Eigenmodes $E_{y}(x)$ for the PCs marked in Figure 8: $\widetilde{\gamma}_{2} \approx 1.3709$ (red, linear case), $\widehat{\gamma}_{1} \approx 1.4548$ (blue), $\widehat{\gamma}_{2} \approx 1.7476$ (green), and $\widehat{\gamma}_{3} \approx 2.7896$ (brown).

therefore the eigenmode. The fact that the power-dependent PCs can have some potential for optical signal processing is pointed out in many papers (see, e.g., [28-34]). For additional description of applications of nonlinear guided waves and Kerr effect, see [32,34], where many useful references are also given.

The result given in the paper clearly shows that nonlinear problems can have solutions that cannot be considered as perturbations of solutions of corresponding linear problems. Thus, it is necessary to be careful when one linearizes a nonlinear problem and considers the linearized problem without proving that there are no other solutions.

Of course, it is impossible to expect the existence of purely nonlinear waves for infinitely many PCs. However, it is possible that purely nonlinear waves can be observed in an experiment for some first purely nonlinear PCs. Indeed, as is seen from Figures 5, 7, and 9, max values of few first purely nonlinear eigenmodes are not too big (in comparison with the linear eigenmode). This probably gives an opportunity to observe such waves in an experiment. For the rest of the PCs, 


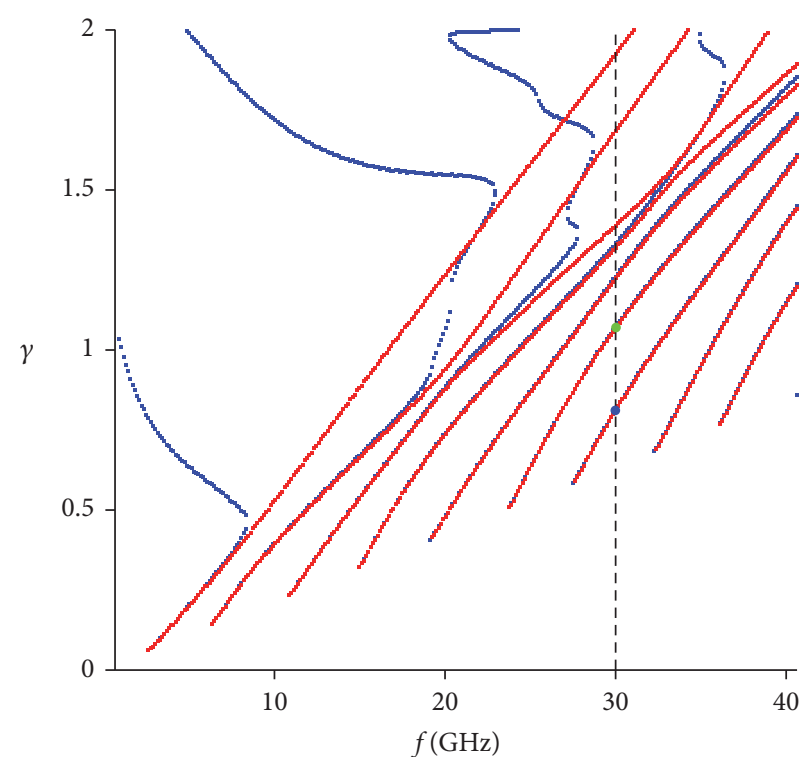

(a)

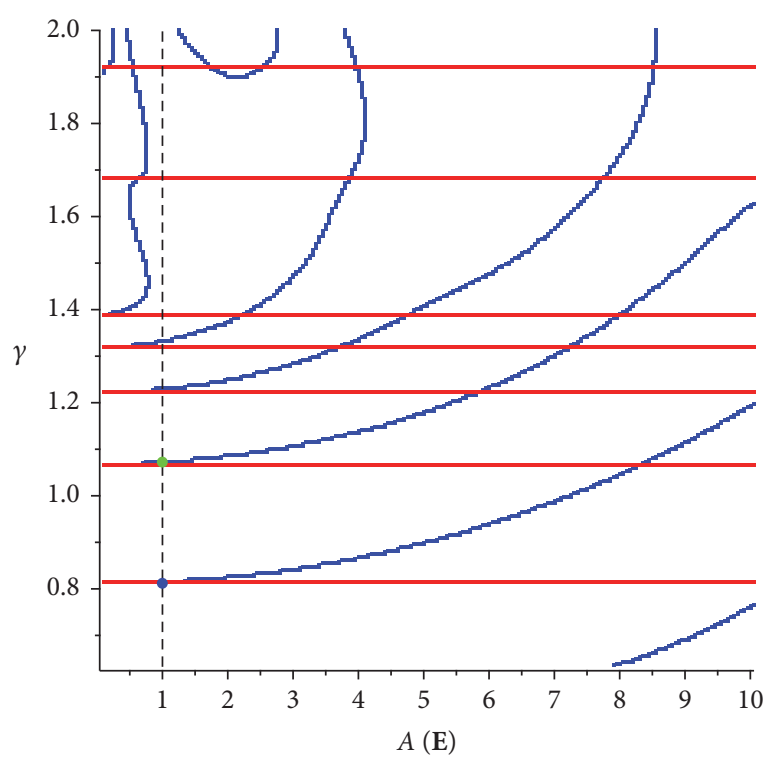

(b)

Figure 10: (a) DCs for nonlinear (blue) and linear (red) cases: $\alpha_{1}=0.02, \alpha_{2}=0.01\left[\mathbf{E}^{-2}\right], h_{1}=10, h_{2}=5[\mathrm{~mm}]$, and $E_{y}(0)=1[\mathbf{E}]$; black dashed line $f=30.2[\mathrm{GHz}]$; nonlinear PCs: $\widehat{\gamma}_{1} \approx 0.8145$ (blue dot) and $\widehat{\gamma}_{2} \approx 1.0723$ (green dot), respectively. (b) The dependence $\gamma$ versus $A$ for nonlinear (blue) and linear (red) cases: $\alpha_{1}=0.02, \alpha_{2}=0.01\left[\mathrm{E}^{-2}\right], h_{1}=10, h_{2}=5[\mathrm{~mm}]$, and $f=30.2[\mathrm{GHz}]$; black dashed line $E_{y}(0)=1[\mathrm{E}]$; nonlinear PCs: $\widehat{\gamma}_{1} \approx 0.8145$ (blue dot) and $\widehat{\gamma}_{2} \approx 1.0723$ (green dot), respectively.
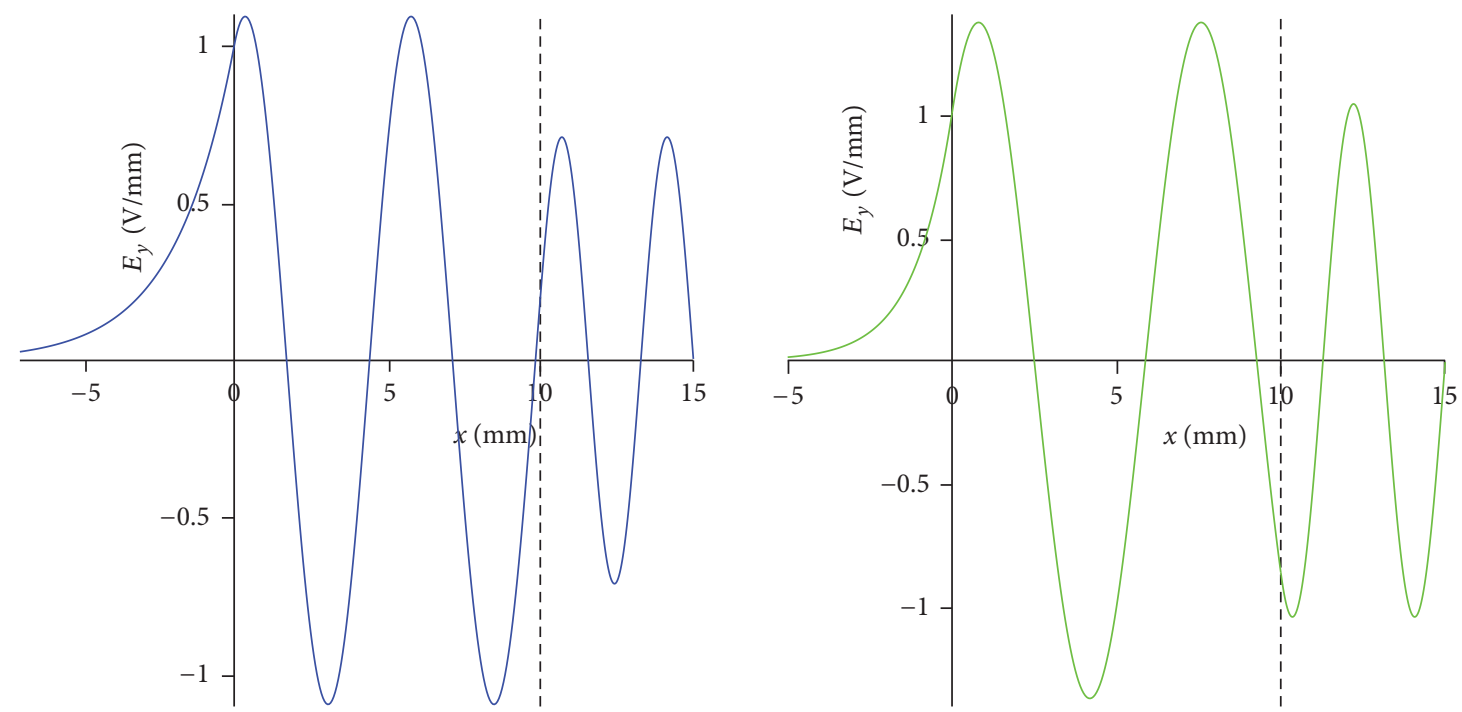

FIGURE 11: Eigenmodes $E_{y}(x)$ for the PCs marked in Figure 10: $\widehat{\gamma}_{1} \approx 0.8145$ (blue) and $\widehat{\gamma}_{2} \approx 1.0723$ (green).

the value $\max _{x \in\left[0, h_{1}+h_{2}\right]}\left|E_{y}\left(x ; \widehat{\gamma}_{i}\right)\right|$ is so high that the Kerr law is no longer valid.

As a matter of fact, different types of nonlinearities admit nonlinear solutions that become linear ones in the linear limit. For example, for a wide range of saturated nonlinearities, there exist only a finite number of PCs $[40,41]$. Thus, there is a qualitative difference between saturated and unbounded (Kerr, qubic-quintic-septic, and, more generally, power and polynomial) nonlinearities. It seems that this difference can be used in order to understand what kinds of nonlinear permittivities are closer to real situations.

If the purely nonlinear guided modes are confirmed by experiment, the theory of nonlinear guided wave propagation will definitely advance. If they are not observed in experiments, then well-known and widespread formulas for nonlinear permittivities must be changed so that mathematical analysis of these models can give results that better satisfy reality. 


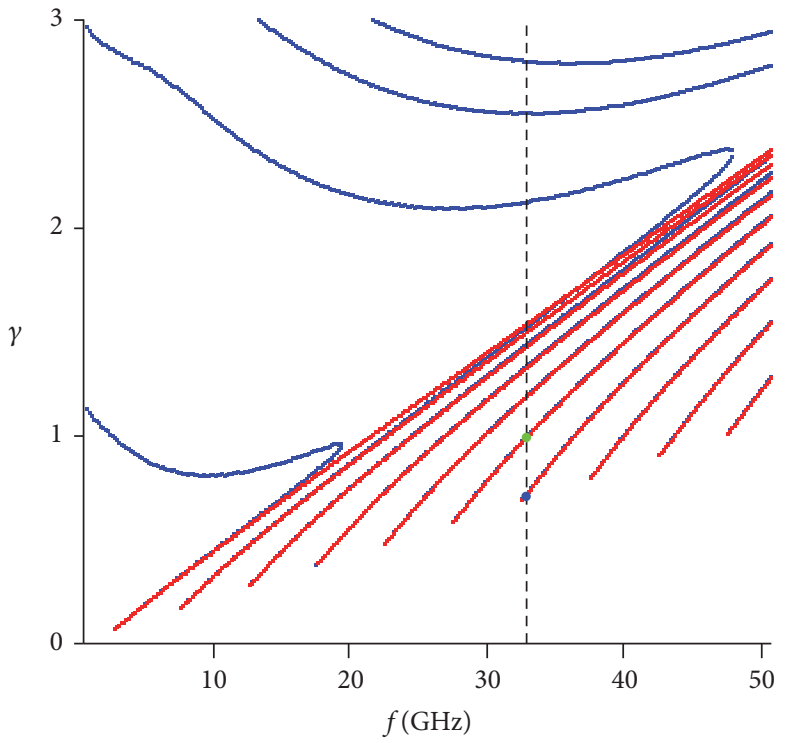

(a)

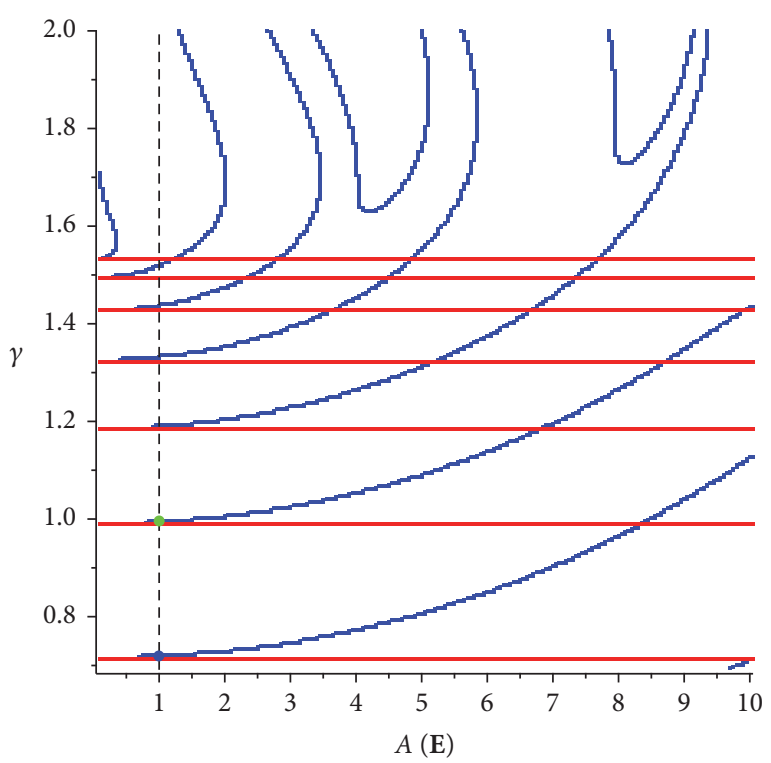

(b)

Figure 12: (a) DCs for nonlinear (blue) and linear (red) cases: $\alpha_{1}=0.02, \alpha_{2}=0.01\left[\mathbf{E}^{-2}\right], h_{1}=10, h_{2}=5[\mathrm{~mm}]$, and $E_{y}(0)=1[\mathrm{E}]$; black dashed line $f=33[\mathrm{GHz}]$; nonlinear PCs: $\widehat{\gamma}_{1} \approx 0.7189$ (blue dot) and $\widehat{\gamma}_{2} \approx 0.9937$ (green dot), respectively. (b) The dependence $\gamma$ versus $A$ for nonlinear (blue) and linear (red) cases: $\alpha_{1}=0.02, \alpha_{2}=0.01\left[\mathbf{E}^{-2}\right], h_{1}=10, h_{2}=5[\mathrm{~mm}]$, and $E_{y}(0)=1[\mathbf{E}]$; black dashed line $f=33[\mathrm{GHz}]$; nonlinear PCs: $\widehat{\gamma}_{1} \approx 0.7189$ (blue dot) and $\widehat{\gamma}_{2} \approx 0.9937$ (green dot), respectively.
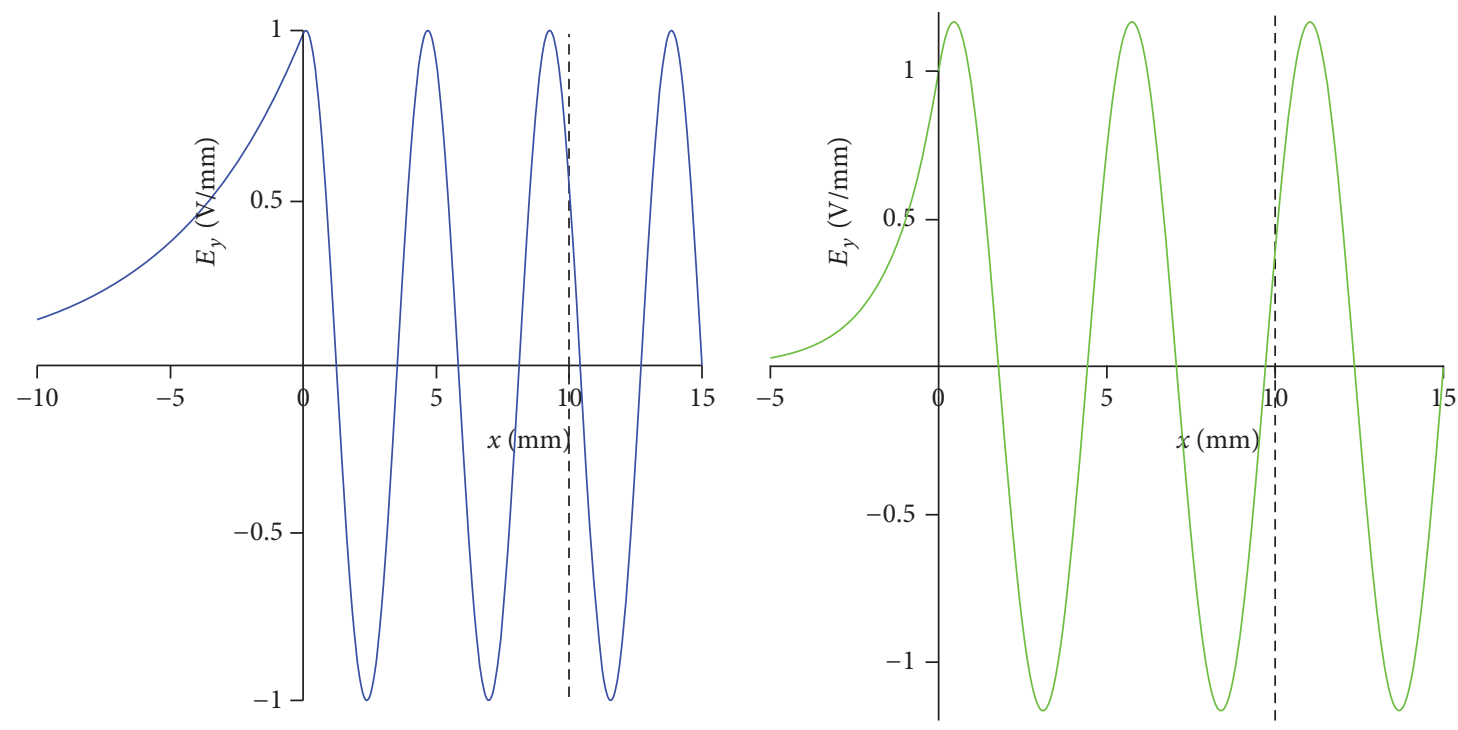

FIgURE 13: Eigenmodes $E_{y}(x)$ for the PCs marked in Figure 12: $\widehat{\gamma}_{1} \approx 0.7189$ (blue) and $\widehat{\gamma}_{2} \approx 0.9937$ (green).

In spite of the fact that in some papers the nonlinear eigenmodes are searched in an explicit form (see, e.g., [7, 16, $17,20-22,34])$, this way is not an appropriate one. Indeed, on one hand, explicit solutions to nonlinear equations are often complicated special functions (if it is possible to find them at all) and, therefore, it is almost impossible to study such solutions. On the other hand, many properties/characteristics of eigenwaves can be calculated from original differential equations and boundary conditions, like it is done in this paper. In order to check calculations, one solves numerically the DE (58) with respect to $\gamma$ in a prescribed segment; then, for each found PC, one solves the Cauchy problem for (7) and (8) with initial data at $x=0$ and transmission conditions (11) at $x=h_{1}$. In this case, the second condition (10) is fulfilled indispensably (obviously, in numerical calculations, $y_{2}\left(h_{1}+h_{2}\right)$ cannot be exactly zero). Thus, Theorems 1,2 , and 3 together with computations allow one to find all nonlinear eigenwaves for which the PCs are calculated. 


\section{Conflicts of Interest}

The authors declare that there are no conflicts of interest regarding the publication of this paper.

\section{Acknowledgments}

This study is supported by the Russian Foundation for Basic Research (Grant no. 15-01-00206), the Russian Federation President Grant (Project no. MK-4684.2016.1), and the Ministry of Education and Science of the Russian Federation (Agreement no. 1.894.2017/4.6).

\section{References}

[1] L. A. Vainstein, Electromagnetic Waves, Radio i svyaz, Moscow, Russia, 1988.

[2] M. J. Adams, An Introduction to Optical Waveguides, John Wiley \& Sons, Chichester, UK, 1981.

[3] A. Snyder and J. Love, Optical Waveguide Theory, Chapman and Hall, London, UK, 1983.

[4] A. S. Zil'bergleit and Y. I. Kopilevich, Spectral Theory of Regular Waveguides, Leningrad, Russia, FTI, 1983.

[5] N. Bloembergen, Nonlinear Optics: A Lecture Note, W. A. Benjamin, Inc., New York, NY, USA, 1965.

[6] S. Kielich, Molecular Nonlinear Optics, PWN, Warszawa, Poland, 1977.

[7] A. Boardman, P. Egan, F. Lederer, U. Langbein, and D. Mihalache, "Third-Order Nonlinear Electromagnetic TE and TM Guided Waves," in Nonlinear Surface Electromagnetic Phenomena, vol. 29 of Modern Problems in Condensed Matter Sciences, pp. 73-287, Elsevier, Amsterdam, The Netherlands, 1991, Reprinted from Nonlinear Surface Electromagnetic Phenomena, H.-E. Ponath and G. I. Stegeman Eds.

[8] B. N. Delone, Basics of Interaction of Laser Radiation with Matter, Editions Frontiéres, Paris, France, 1993.

[9] Y. R. Shen, The Principles of Nonlinear Optics, John Wiley \& Sons, New York, NY, USA, 1984.

[10] N. N. Akhmediev and A. Ankevich, Solitons, Nonlinear Pulses and Beams, Chapman and Hall, London, UK, 1997.

[11] R. W. Boyd, Nonlinear Optics, Academic Press, New York, NY, USA, 2nd edition, 2003.

[12] G. P. Agrawal, Nonlinear Fiber Optics, Academic Press, 1989.

[13] D. N. Klyshko, Photons and Nonlinear Optics, Gordon and Breach Science, 1988.

[14] P. N. Eleonskii, L. G. Oganes'yants, and V. P. Silin, "Cylindrical nonlinear waveguides," Soviet Physics JETP, vol. 35, no. 1, pp. 44-47, 1972.

[15] R. I. Joseph and D. N. Christodoulides, "Exact field decomposition for TM waves in nonlinear media," Optics Expresss, vol. 12, no. 10, pp. 826-828, 1987.

[16] D. Mihalache, R. G. Nazmitdinov, and V. K. Fedyanin, "Nonlinear optical waves in layered structures," Physics of Elementary Particles and Atomic Nuclei, vol. 20, no. 1, pp. 198-253, 1989 (Russian).

[17] D. Mihalache, R. G. Nazmitdinov, V. K. Fedyanin, and R. P. Wang, "Nonlinear guided waves in planar structures," Physics of Elementary Particles and Atomic Nuclei, vol. 23, no. 1, pp. 122173, 1992 (Russian).
[18] A. D. Boardman and P. Egan, "Novel nonlinear surface and guided TE waves in asymmetric LHM waveguides," Journal of Optics A: Pure and Applied Optics, vol. 11, no. 11, Article ID 114032, 2009.

[19] A. D. Boardman, M. M. Shabat, and R. F. Wallis, "Nonlinear magnetodynamic waves on magnetic materials," Physical Review B: Condensed Matter and Materials Physics, vol. 41, no. 1, pp. 717-730, 1990.

[20] H. W. Schürmann, "On the theory of TE-polarized waves guided by a nonlinear three-layer structure," Zeitschrift für Physik B Condensed Matter, vol. 97, no. 4, pp. 515-522, 1995.

[21] H. W. Schürmann, V. S. Serov, and Y. V. Shestopalov, "Solutions to the Helmholtz equation for TE-guided waves in a threelayer structure with Kerr-type nonlinearity," Journal of Physics A: Mathematical and General, vol. 35, no. 50, pp. 10789-10801, 2002.

[22] H. W. Schürmann and V. Serov, "Theory of TE-polarized waves in a lossless cubic-quintic nonlinear planar waveguide," Physical Review A: Atomic, Molecular and Optical Physics, vol. 93, no. 6, Article ID 063802, 2016.

[23] Y. G. Smirnov and D. V. Valovik, "Guided electromagnetic waves propagating in a plane dielectric waveguide with nonlinear permittivity," Physical Review A: Atomic, Molecular and Optical Physics, vol. 91, no. 1, Article ID 013840, 6 pages, 2015.

[24] Y. G. Smirnov and D. V. Valovik, "On the infinitely many nonperturbative solutions in a transmission eigenvalue problem for Maxwell's equations with cubic nonlinearity," Journal of Mathematical Physics, vol. 57, no. 10, Article ID 103504, 15 pages, 2016.

[25] D. Valovik, "Novel propagation regimes for TE waves guided by a waveguide filled with Kerr medium," Journal of Nonlinear Optical Physics \& Materials, vol. 25, no. 4, Article ID 1650051, 17 pages, 2016.

[26] A. Yariv and P. Yeh, "Electromagnetic propagation in periodic stratified media. ii. birefringence, phase matching, and x-ray lasers," The Optical Society of America, vol. 67, no. 4, pp. 438448, 1977.

[27] P. Yeh, A. Yariv, and C. S. Hong, "Electromagnetic propagation in periodic stratified media. i. general theory," The Optical Society of America, vol. 67, no. 4, pp. 423-438, 1977.

[28] U. Trutschel, F. Lederer, and M. Golz, "Nonlinear guided waves in multilayer systems," IEEE Journal of Quantum Electronics, vol. 25, no. 2, pp. 194-200, 1989.

[29] W. Biehlig, F. Lederer, U. Trutschel, U. Langbein, D. Mihalache, and A. D. Boardman, "TM-Polarized Nonlinear Guided Waves in Multilayer Systems," IEEE Journal of Quantum Electronics, vol. 27, no. 2, pp. 238-242, 1991.

[30] W. Biehlig, F. Lederer, and U. Trutschel, "An Effective Medium Model for TM-Polarized Nonlinear Guided Waves in Multilayer Systems," IEEE Journal of Quantum Electronics, vol. 28, no. 7, pp. 1643-1646, 1992.

[31] L.-P. Yuan and L.-P. Yuan, "A Unified Approach for the CoupledMode Analysis of Nonlinear Optical Couplers," IEEE Journal of Quantum Electronics, vol. 30, no. 1, pp. 126-133, 1994.

[32] J. D. Begin and M. Cada, "Exact Analytic Solutions to the Nonlinear Wave Equation for a Saturable Kerr-Like Mediur: Modes of Nonlinear Optical Waveguides and Couplers," IEEE Journal of Quantum Electronics, vol. 30, no. 12, pp. 3006-3016, 1994.

[33] J.-G. Ma and Z. Chen, "Numerically determining the dispersion relations of nonlinear TE slab-guided waves in non-kerr-like media," IEEE Transactions on Microwave Theory and Techniques, vol. 45, no. 7, pp. 1113-1117, 1997. 
[34] Y.-D. Wu and M.-H. Chen, "Method for analyzing multilayer nonlinear optical waveguide," Optics Express, vol. 13, no. 20, pp. 7982-7996, 2005.

[35] P. J. Torres, "Guided waves in a multi-layered optical structure," Nonlinearity, vol. 19, no. 9, pp. 2103-2113, 2006.

[36] D. V. Valovik, "Integral dispersion equation method to solve a nonlinear boundary eigenvalue problem," Nonlinear Analysis: Real World Applications, vol. 20, pp. 52-58, 2014.

[37] I. G. Petrovsky, Lectures on the Theory Ordinary Differential Equations, Moscow State University, Moscow, Russia, 1984.

[38] Yu. N. Bibikov, Course of Ordinary Differential Equations, Moscow Vysshaya Shkola, Moscow, Russia, 1991.

[39] D. Valovik, "On the existence of infinitely many nonperturbative solutions in a transmission eigenvalue problem for nonlinear Helmholtz equation with polynomial nonlinearity," Applied Mathematical Modelling, vol. 53, pp. 296-309, 2018.

[40] D. V. Valovik, "Nonlinear coupled electromagnetic wave propagation: saturable nonlinearities," Wave Motion. An International Journal Reporting Research on Wave Phenomena, vol. 60, pp. 166-180, 2016.

[41] D. V. Valovik and V. Y. Kurseeva, "On the eigenvalues of a nonlinear spectral problem," Journal of Differential Equations, vol. 52, no. 2, pp. 149-156, 2016. 


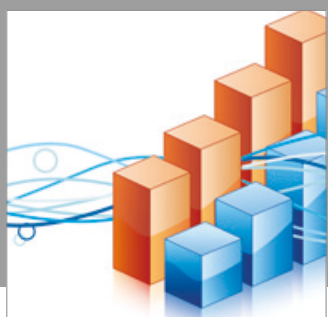

Advances in

Operations Research

vatersals

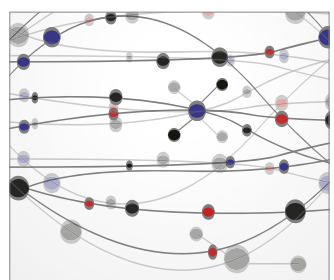

\section{The Scientific} World Journal
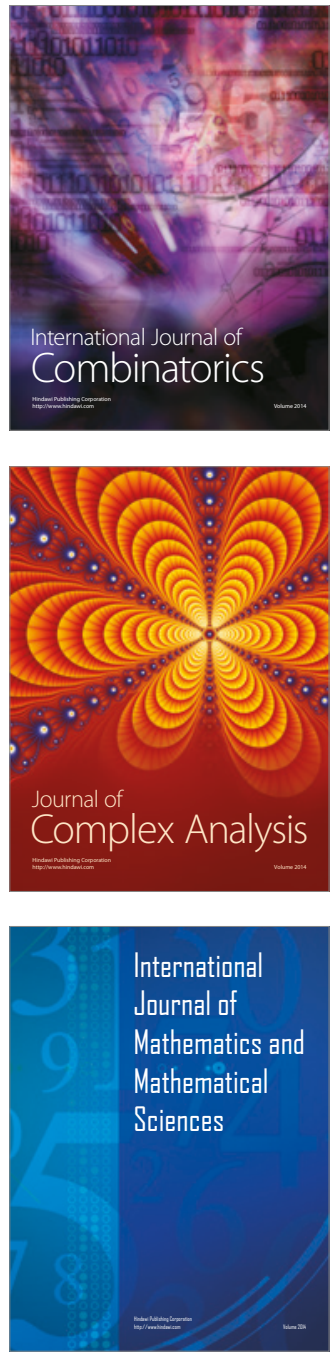
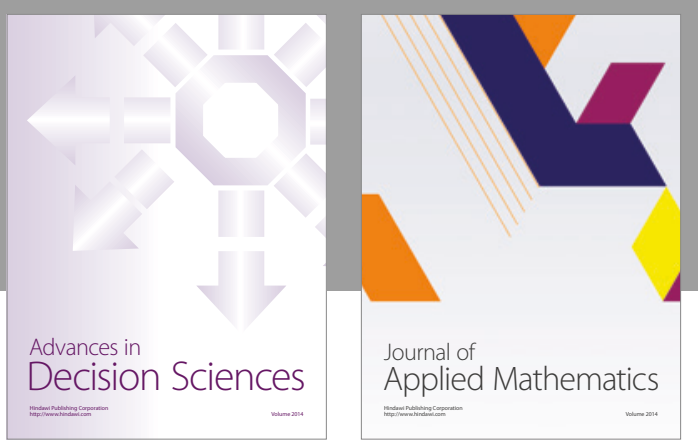

Algebra

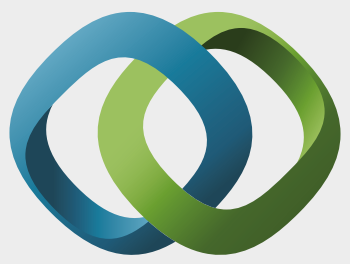

\section{Hindawi}

Submit your manuscripts at

https://www.hindawi.com
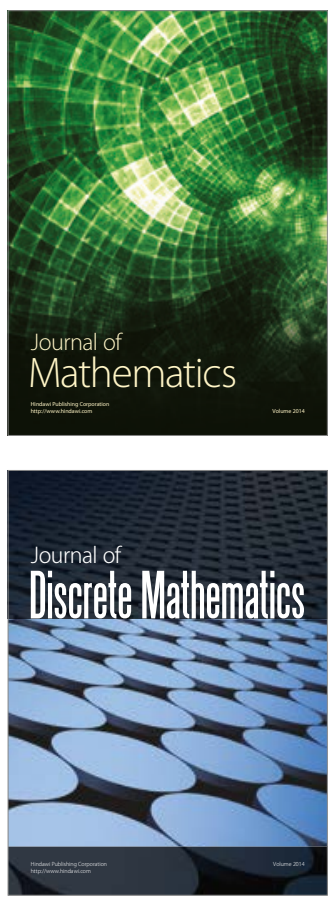

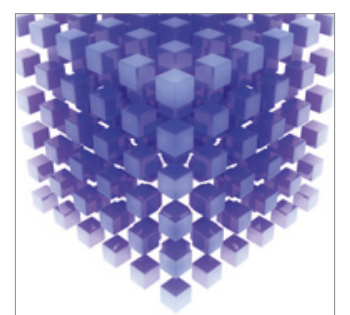

Mathematical Problems in Engineering
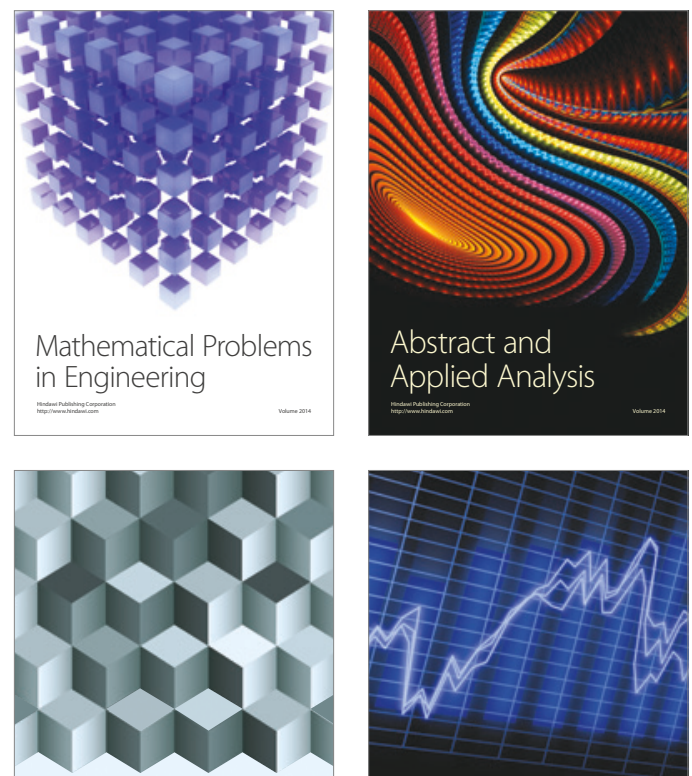

Journal of

Function Spaces

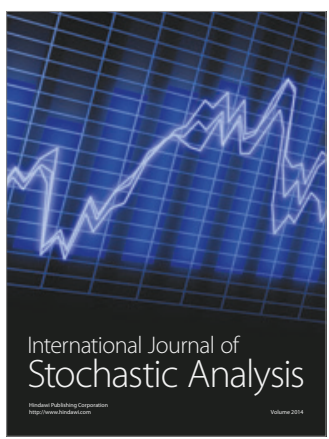

Probability and Statistics
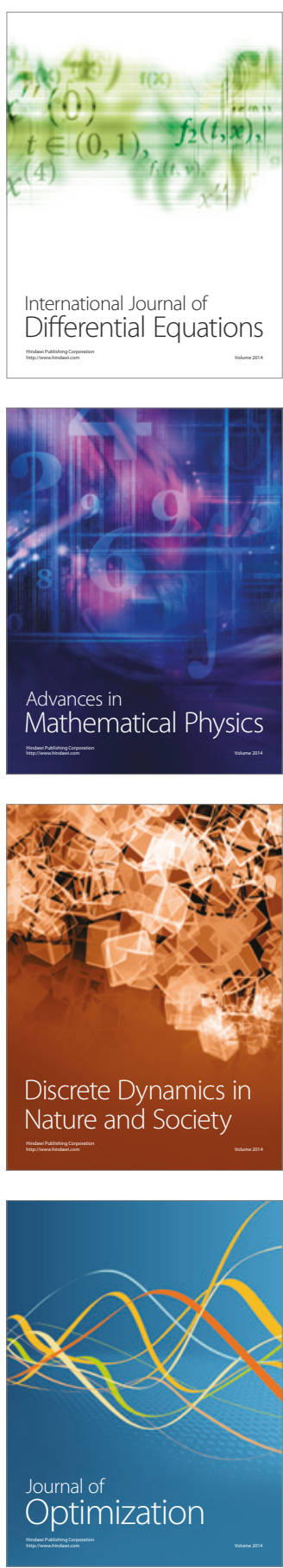